Egypt. Acad. J. Biolog. Sci., 13(2):1-34 (2020)

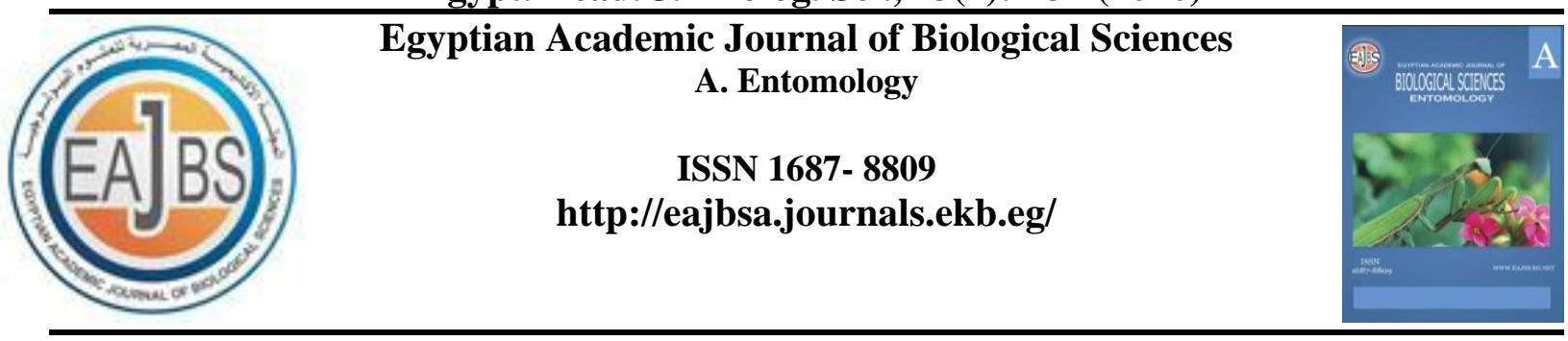

\title{
Revision of family Noctuidae (4) \\ Subfamilies "Bryophilinae, Condicinae, Cuculliinae, Eriopinae and Eustrotiinae" of Egypt (Lepidoptera, Noctuidae)
}

\author{
Abdelfattah, M. A. Salem \\ Senior Scientific Officer, Inter African Phytosanitary Council, African Union \\ Email: AmerA@africa-union.org; abdelfattahsalem@ymail.com
}

\begin{abstract}
ARTICLE INFO
Article History

Received:20/3/2020

Accepted:10/4/2020

Keywords:

Lepidoptera,

Noctuidae,

Bryophilinae,

Condicinae,

Cuculliinae,

Eriopinae

Eustrotiinae,

\section{ABSTRACT}

This is the last paper on the revision of Noctuidae in Egypt, we revised 11 subfamilies (from total 16 subfamilies). In this study, we are exposed to revise the last five subfamilies which are Bryophilinae, Condicinae, Cuculliinae, Eriopinae, and Eustrotiinae. These subfamilies contain 40 species under 10 genera. Subfamily Eustrotiinae Grote, 1882 is the largest subfamily (in this study) with 11 species and 3 genera, followed by Subfamily Bryophilinae Guenée, 1852 with 11 species under one genera while Subfamily Eriopinae Herrich-Schäffer, [1851] is the smallest subfamily containing two species under one genus.

We added three Annexes, one for the recent situation of Family Noctuidae; $2^{\text {nd }}$ a list of noctuid types in Egypt and the $3^{\text {rd }}$ is the complete recent list of family Noctuidae in Egypt.
\end{abstract}

Egypt

\section{INTRODUCTION}

This is the last paper in the series revision of the Noctuidae of Egypt ( $4^{\text {th }}$ paper). During this study, we revised the last 5 subfamilies (Bryophilinae, Condicinae, Cuculliinae, Eriopinae, and Eustrotiinae).

Based on material from the main reference collections in Egypt, light traps collections, National museums, and other references, 40 species in the 5 subfamilies (Bryophilinae, Condicinae, Cuculliinae, Eriopinae, and Eustrotiinae) under 10 genera were listed. Some further taxonomic changed re-proposed. Taxonomic position, synonyms, and types are included and photographs for available species are provided.

Bryophilinae is a big subfamily with about 200 species worldwide (Speidel et al., 1996a); most species are small, and their larvae feed on lichens and rest well concealed in holes and crevices during the daytime, feeding at night, with their food becoming softened by nocturnal humidity. The larvae pupate in a spun cocoon in the shelter of holes and ledges. The coloration of larvae is non-protective, but that of the adult remarkably matches the different hues of the lichen-covered surface on which they rest (Seitz, 1914).

Genus Cryphia was the only genus recorded in Egypt with 11 species.

Condicinae was once included in the polyphyletic Amphipyrinae. In Europe, they are represented by four genera: Condica Walker, 1856, Hadjina Staudinger, 1892, Acosmetia 
Stephens, 1829 and Eucarta Lederer, 1857 (Fibiger \& Hacker, 2005). So far, 6 species belonging to 2 genera under one tripe have been recorded in Egypt

Cuculliinae is a large and phylogenetically compact subfamily with more than 200 species worldwide (Speidel et al., 1996a). Most species are xerophilous, inhabiting steppes, eremic areas and/or xeromontane habitats (Ronkay \& Ronkay 1994, 1995). They are usually quite attracted to ultraviolet light, but often not before midnight.

Ten species belonging to 3 genera under one tribe were recorded in Egypt

Eriopinae was previously either placed in Amphipyrinae, Hadeninae, or its own subfamily Eriopinae. Due to the complicated taxonomic histories of all the trifine groups of the Noctuidae sensu lato during the last decades, and the varying intensities of the evidence used for inferring the phylogenetic relationships, no consensus about the systematic position of the Eriopinae has ever been reached.

So far, 2 species have been recorded in Egypt belonging to one genera.

Eustrotiinae were often included in the Acontiinae, but interpreted by Fibiger \& Lafontaine (2005) as an independent subfamily. The systematic scope is very uncertain. The genusgroup of Eublemma is included in the database "Butterflies and Moths of the World - Generic Names and their Type-species" of the Natural History Museum London. This group is described by Kravchenko et al. (2007) considered as a separate subfamily Eublemminae. The Eustrotiinae are probably paraphyletic.

Up to date, 11 species were recorded in Egypt under 3 genera.

\section{MATERIALS AND METHODS}

The present work based upon:

* The main five reference insect collections in Egypt: Collection of Ministry of Agriculture, Plant Protection Research Institute (PPRI); Collection of Faculty of Science, Cairo University (CUC); Collection of Faculty of Science, Ein Shams University (ESUC); Collection of El Alfieri, Al Azhar University, Faculty of Agriculture (ALUC) and Collection of Egyptian Entomological Society (EESC).

* The published papers and Thesis in Egypt.

* Data from the Collections of International Museums (BMNH, London; USNM, Smithsonian, USA; ZI, Leningrad; MNHU, Berlin; MNHN, Paris, ...etc.)

* Main monographs of Lepidoptera in the world

Recent taxonomic position, senior synonyms, types, and host records together with local and world distribution for each species are presented. Photos for all species are also provided (as possible). Tribes, genera, and species are alphabetically arranged.

\section{ACRONOMY}

BMNH: The Natural History Museum, London, United Kingdom

HNHM: Hungarian Natural History Museum, Budapest, Hungary

LNK: Landessammlungen für Naturkunde, Karlsruhe, Germany

LS: Linnean Society, London, United Kingdom

MNGH: National Museum of Natural History Grigore Antipa, Bucharest, Romania

MNHN: Muséum National d'Histoire Naturelle, Paris, France

MNHU: Museum für Naturkunde der Humboldt Universität, Berlin, Germany

NHMW: Naturhistorisches Museum Wien, Vienna, Austria

NHRM: Naturhistoriska Riksmuseet, Stockholm, Sweden

NM: National Museum in Prague

USNM: National Museum of Natural History, Smithsonian Institution, Washington, U.S.A.

ZI: Zoological Institute, Academy of Sciences, Leningrad, Russia

ZSM: Zoologische Staatssammlung, Münchhausenstr. Munich, Germany 
ZMHB: Zoologisches Institut und Museum, Universität Hamburg, Germany.

ZMUC: Zoologisk Museum, Universitetsparken, Copenhagen, Denmark

\section{RESULTS AND DISCUSSION}

The revision of the last list of Noctouidea in Egypt (Amer \& El-Torkey, 2017); indicate that there are few changes:

* Prospalta coptica Wiltshire, 1948 is a synonomy to Condica pauperata (Walker, 1858)

(Condicinae)

* Both Cucullia syrtana (Mabille, 1888) and Cucullia verbasci Linnaeus, 1758 transfer to genus Shargacucullia (Cuculliinae)

* We recorded 11 types (or type synonymy) from Egypt as follows:

\section{Subfamily Bryophilinae Guenée, 1852}

* Cryphia minima Waltshire, 1949 from Wadi Hoff (Cairo, Lower Egypt)

* Cryphia pallida (Bethune-Baker, 1894) from Alexandria (Coastal Strip); (Type: $\mathrm{BMNH}$ )

\section{Subfamily Condicinae Poole, 1995}

* Acosmetia arida Joannis, 1909 from Mokattam (Cairo, Lower Egypt); (Type: MNHN)

* Condica pauperata (Walker, 1858) as Prospalta coptica Wiltshire, 1948 (Marsa Halaib, Gebel Elba)

\section{Subfamily Cuculliinae Herrich-Schäffer, [1850]}

* Cucullia hemidiaphana Graeser, 1892 as Cucullia efflatouni Wiltshire, 1948 from Wadi el Lega, Um Arab, Farsh el Arab (Sinai); (Type: Alfieri Collection, Destroyed); (Paratype: BMNH)

* Cucullia macara Rebel, 1947 from Wadi Cansisrob (Gebel Elba); (Syntype: NHMW)

Subfamily Eustrotiinae Grote, 1882

* Eulocastra alfierii (Wiltshire, 1948) from Bir Isla, Wadi Isla, Karm Alam, (Sinai); (Holotype: USNM)

* Ozarba capreolana Rebel, 1947 from Mersa Halaib (Gebel Elba)

* Ozarba fuscescens Rebel, 1947 from Wadi Eideb (Gebel Elba)

* Ozarba socotrana Hampson, 1910 as Ozarba cervina Rebel, 1948 from Wadi Eideb (Gebel Elba)

* Ozarba variabilis Berio, 1940 as Ozarba elephina Rebel, 1948 from Wadi Eideb (Gebel Elba)

There are 3 species recorded only from Egypt: Acosmetia arida Joannis, 1909

(Condicinae); Ozarba capreolana Rebel, 1947 (Eustrotiinae) and Ozarba fuscescens Rebel, 1947 (Eustrotiinae)

\section{List of subfamilies, genera and species:}

Subfamily: Bryophilinae Guenée, 1852

1. Cryphia Hübner, 1818

1. Cryphia algae (Fabricius, 1775)

2. Cryphia amasina (Drandt, 1931)

3. Cryphia fraudatricula (Hübner, 1803)

4. Cryphia maeonis (Lederer, 1865)

5. Cryphia minima Waltshire, 1949

6. Cryphia pallida (Bethune-Baker, 1894)

7. Cryphia paulina (Staudinger, 1892)

8. Cryphia petrea (Guenée, 1852)

9. Cryphia raptricula (Denis \& Schiffermüller, 1775)

10. Cryphia receptricula (Hübner, 1803)

11. Cryphia vandalusiae (Duponchel, 1842) 
Subfamily: Condicinae Poole, 1995

Tribe: Condicini Poole, 1995

2. Acosmetia Stephens, 1829

12. Acosmetia arida Joannis, 1909

3. Condica Walker, 1856

13. Condica capensis (Guenée, 1852)

14. Condica conducta (Walker, 1857)

15. Condica palaestinensis (Staudinger, 1895)

16. Condica pauperata (Walker, 1858)

17. Condica viscosa (Freyer, 1831)

Subfamily Cuculliinae Herrich-Schäffer, [1850]

Tribe Cucullini Ronkay \& Gyulai, 2011

4. Cucullia Schrank, 1802

18. Cucullia calendula Treitschke, 1835

19. Cucullia chamomillae (Denis \& Schiffermüller, 1775)

20. Cucullia hemidiaphana Graeser, 1892

21. Cucullia macara Rebel, 1947

22. Cucullia melanoglossa (Berio, 1934)

23. Cucullia santolinae Rambur, 1834

24. Cucullia syrtana (Mabille, 1888)

5. Shargacucullia Ronkay \& Ronkay, 1992

25. Shargacucullia strigicosta (Boursin, 1940)

26. Shargacucullia verbasci Linnaeus, 1758

6. Metlaouia Dumont, 1929

27. Metlaouia autumna (Chrétien, 1910)

Subfamily Eriopinae Herrich-Schäffer, [1851]

7. Callopistria Hubner, [1821]

28. Callopistria latreillei (Duponchel, 1827)

29. Callopistria yerburii (Butler, 1884)

Subfamily Eustrotiinae Grote, 1882

8. Eulocastra Butler, 1886

30. Eulocastra alfierii (Wiltshire, 1948)

31. Eulocastra diaphora (Staudinger, 1878)

32. Eulocastra platizona (Lederer, 1869)

33. Eulocastra rex (Wiltshire, 1948)

34. Eulocastra tamsina Brandt, 1947

8. Ozarba Walker, 1865

35. Ozarba capreolana Rebel, 1947

36. Ozarba fuscescens Rebel, 1947

37. Ozarba scotrana Berio, 1940

38. Ozarba variabilis Berio, 1940

9. Pseudozarba Warren, 1913

39. Pseudozarba bipartita (Herrich-Schäffer, 1850)

40. Pseudozarba menozona (Hampson, 1910)

Subfamily: Bryophilinae Guenée, 1852 (Plate I)

Bryophilinae is a big subfamily with about 200 species worldwide (Speidel et al., 1996a); most species are small, and their larvae feed on lichens and rest well concealed in holes and crevices during the daytime, feeding at night, with their food becoming softened by nocturnal humidity. The larvae pupate in a spun cocoon in the shelter of holes and ledges. 
The coloration of larvae is non-protective, but that of the adult remarkably matches the different hues of the lichen-covered surface on which they rest (Seitz, 1914).

Genus Cryphia was the only genus recorded in Egypt with 11 species, two of them (Cryphia minima Waltshire, 1949 and Cryphia pallida (Bethune-Baker, 1894) are Egyptian types. Cryphia minima Waltshire, 1949 recorded only in Egypt.

Subfamily Bryophilinae Guenée, 1852

1. Cryphia Hübner, 1818

1. Cryphia algae (Fabricius, 1775)

2. Cryphia amasina (Drandt, 1931)

3. Cryphia fraudatricula (Hübner, 1803)

4. Cryphia maeonis (Lederer, 1865)

5. Cryphia minima Waltshire, 1949

6. Cryphia pallida (Bethune-Baker, 1894)

7. Cryphia paulina (Staudinger, 1892)

8. Cryphia petrea (Guenée, 1852)

9. Cryphia raptricula (Denis \& Schiffermüller, 1775)

10. Cryphia receptricula (Hübner, 1803)

11. Cryphia vandalusiae (Duponchel, 1842)

\section{Cryphia Hübner, 1818}

1. Cryphia algae (Fabricius, 1775)

Noctua spoliatricula (Denis \& Schiffermüller, 1775)

Noctua degener (Denis \& Schiffermüller, 1775)

Phalaena Noctua chloris (Borkhausen, 1792)

Noctua algae (FABRICIUS, 1775)

Phalaena algae (FABRICIUS, 1775)

Bryophila algae (FABRICIUS, 1775)

Metachrostis algae (FABRICIUS, 1775)

Euthales algae (FABRICIUS, 1775)

Phalaena Noctua calligrapha (BORKHAUSEN, 1792)

Noctua mendacula (HÜBNER, 1813)

Common name: Tree-lichen Beauty

Wingspan: $24-30 \mathrm{~mm}$

Host records: Tree lichen

Geographical Records: Albania, Algeria, Armenia, Austria, Belgium, Bulgaria, Corsica, Cyprus, Czech Republic, Denmark, England, Finland, France, Germany, Greece, Hungary, Iran, Iraq, Israel, Italy, Libya, Lithuania, Malta, Morocco, Netherlands, Palestine, Poland, Portugal, Romania, Russia, Sardinia, Sicily, Slovakia, Spain, Sweden, Switzerland, Tunisia, Turkey, Ukraine, Yugoslavia

Distribution in Egypt: Coastal Stripe

Type Locality: Germany

Type specimen:

Type: Germany

2. Cryphia amasina (Drandt, 1931)

Bryophila muralis ssp. amasina Draudt, 1931

Common name: Unknown

Wingspan: $26-29 \mathrm{~mm}$

Host records: Lichens

Geographical Records: Albania, Bulgaria, Croatia, Greece, Jordan, Israel, Lebanon, Romania, Saudi Arabia, Turkmenistan, Turkey, Yemen

Distribution in Egypt: Alexandria, Mariout (Coastal Stripe) 
Type Locality: Turkey, Amasia

Type specimen:

Syntype: Turkey, Amasia

3. Cryphia fraudatricula (Hübner, 1803)

Noctua fraudatricula Hübner, [1803]

Common name: Unknown

Wingspan: about $32 \mathrm{~mm}$.

Host records: Lichens

Geographical Records: Albania, Austria, Bulgaria, Czech Republic, Finland, France, Germany, Greece, Hungary, Italy, Japan, Lithuania, Poland, Romania, Russia, Slovakia, Spain, Switzerland, Ukraine, Yugoslavia

Distribution in Egypt: Coastal Stripe

Type Locality: Europa

Type specimen:

Type: Europe

4. Cryphia maeonis (Lederer, 1865)

Cryphia praecana Christoph, 1893

Cryphia rubellina Staudinger, 1900

Metachrostis sabulosa (Warren, 1909)

Bryophila rubrolineata (Osthelder, 1933)

Criphia sabulicolor (deLattin, 1950)

Common name: Unknown

Wingspan: $29-34 \mathrm{~mm}$

Host records: Lichens

Geographical Records: Afghanistan, Armenia, Cyprus, Greece, Iran, Iraq, Israel, Jordan,

Palestine, Syria, Turkey, Turkmenistan

Distribution in Egypt: Eastern desert

Type locality: Turkey, Anatolia

Type specimens:

Syntype: MNHU, Berlin

5. Cryphia minima Waltshire, 1949

Bryoleuca minima (Wiltshire, 1949)

Common name: Unknown

Wingspan: $21-25 \mathrm{~mm}$.

Host records: Lichens

Geographical Records: Israel, Palestine, Saudi Arabia

Distribution in Egypt: Lower Egypt (Wadi Hoff).

Type Locality: Egypt, Cairo (Wadi Hoff, Ougret El-Sheikh)

Common name: Unknown

6. Cryphia pallida (Bethune-Baker, 1894)

Wingspan: 19- $22 \mathrm{~mm}$

Host records: Lichens

Geographical Records: Algeria, France, Italy, Malta, Morocco, Portugal, Sardinia, Spain

Distribution in Egypt: Coastal Stripe (Alexandria, Mariout).

Type locality: Egypt, Alexandria

Type specimens:

Type: BMNH, London

Caradrina keltana Amsel. 1935

7. Cryphia Paulina (Staudinger, 1891)

Cryphia paulina subsp. fuscirena Wiltshire, 1980 
Cryphia paulina subsp. mizbilensis Wiltshire, 1980

Common name: Unknown

Wingspan: $19-22 \mathrm{~mm}$

Host records: Lichens

Geographical Records: Israel, Jordan, Palestine, Saudi Arabia

Distribution in Egypt: Sinai

Type locality: Palestine/Israel, near Jerusalem

Type specimens:

Syntype: MNHU (ZMHB), Berlin

8. Cryphia petrea (Guenée, 1852)

Bryophila ramosana (Draudt, 1931)

Bryophila transversa (Draudt, 1931)

Common name:

Wingspan: $26-30 \mathrm{~mm}$

Host records: Lichens

Geographical Records: Crete, Croatia, Cyprus, France, Greece, Israel, Lebanon, Morocco, Palestine, Portugal, Sicily, Spain, Turkey

Distribution in Egypt: Eastern desert

Type locality: Spain, Andalusia

9. Cryphia raptricula (Denis \& Schiffermüller, 1775)

Noctua raptricula Denis \& Schiffermüller, 1775

Phalaena divisa Esper, 1791

Phalaena palliola Borkhausen, 1792

Phalaena pomula Borkhausen, 1792

Noctua deceptricula Hübner, [1803]

Bryophila carbonis Freyer, 1849

Bryophila oxybiensis Millière, 1875

Bryophila raptriculoides Turati, 1912

Catamecia bryophiloides Rothschild, 1914

Bryophila dolopis f. pallidior Draudt, 1931

Bryophila dolopis f. striata Draudt, 1931

Common name: Marbled Grey

Wingspan: $27-35 \mathrm{~mm}$

Host records: Lichens, Parmelia centrifuga, P. saxatilis.

Geographical Records: Afghanistan, Algeria, Armenia, Ashkhabad, Austria, China, England, France, Germany, Hungary, Iran, Iraq, Israel, Italy, Korea, Lebanon, Malta, Morocco, Portugal, Sardinia, Spain, Syria, Ukraine, USSR

Distribution in Egypt: Coastal Stripe (Kingi Mariout)

Type locality: Austria, Vienna

Type specimens:

Type: Destroyed

10. Cryphia receptricula (Hübner, 1803)

Noctua strigula (Borkhausen, 1792)

Bryophila stictica (Ménétriés, 1859

Common name: Unknown

Wingspan: $20-24 \mathrm{~mm}$

Host records: Lichens, Parmelia centrifuga, P. saxatilis.

Geographical Records: Armenia, Austria, Bulgaria, Czech Republic, Cyprus, Greece, Germany, Hungary, Italy, Iran, Palestine, Poland, Romania, Russia, Sicily, Slovakia, Switzerland, Syria, Turkey, Ukraine, Yugoslavia 
Distribution in Egypt: Coastal Stripe

Type locality: Europe

11. Cryphia vandalusiae (Duponchel, 1842)

Miana trilinea Bethune-Baker, 1894; Type: Egypt, Alexandria

Bryophila vandalusiae Duponchel, 1842

Cryphia trilinea Bethune-Baker, 1894

Metachrostis syrticiola Turati, 1924

Cryphia rectilinea auberti Boursin, 1952

Bryoleuca trilinea Hampson, 1908

Common name: Unknown

Wingspan: $20-23 \mathrm{~mm}$

Host records: Lichens

Geographical Records: Corsica, France, Libya, Morocco, Portugal, Italy, Sardinia, Spain

Distribution in Egypt: Coastal Stripe (Alex.).

Type locality: Spain, Andalusia

Type specimens:

Type: Spain, Andalusia

\section{Subfamily: Condicinae Poole, 1995 (Plate I)}

The subfamily Condicinae was once included in the polyphyletic Amphipyrinae. In Egypt, they are represented by two genera: Condica Walker, 1856, and, Acosmetia Stephens, 1829. So far, 6 species belonging to 2 genera under one tripe have been recorded in Egypt. There are 2 types from Egypt, one of them is recorded only in Egypt from Al-Mokattam (Near Cairo, Lower Egypt).

Subfamily: Condicinae Poole, 1995

Tribe: Condicini

1. Acosmetia Stephens, 1829

1. Acosmetia arida Joannis, 1909

2. Condica Walker, 1856

2. Condica capensis (Guenée, 1852)

3. Condica conducta (Walker, 1857)

4. Condica palaestinensis (Staudinger, 1895)

5. Condica pauperata (Walker, 1858)

6. Condica viscosa (Freyer, 1831)

Tribe: Condicini Poole, 1995

1. Acosmetia Stephens, 1829

1. Acosmetia arida Joannis, 1909

Common name: Unknown

Wingspan: about $18 \mathrm{~mm}$.

Host records: Grasses

Geographical Records: Not recorded

Distribution in Egypt: Lower Egypt (Cairo, Mokattam)

Type locality: Egypt (Cairo, Mokattam)

Type specimens:

Syntype: 2 Females (MNHN, Paris)

2. Condica Walker, 1856

1. Condica capensis (Guenée, 1852)

Prospalta capensis Guenée, 1852

Condica afra (Guenée, 1852)

Condica prodita Walker, 1862 
Condica inexacta Walker, 1862

Condica canorufa Walker, 1865

Condica leonina Walker, 1865

Apamea capensis Guenée, 1852

Common name: Unknown

Wingspan: $27-34 \mathrm{~mm}$

Host records: Grasses, oilseed crops (Guizatia sp.) Ageratum, Bidenspilosa, Calendula,

Cinerana, Coreopsis, Cynara, Macadamia

Geographical Records: Australia, Cape Verde, China, Comoros, DRCongo, England, Equatorial Guinea, Eritrea, Ethiopia, Ghana, India, Indonesia, Israel, Jordan, Kenya, Madagascar, Malawi, Mauritania, Morocco, Oman, Pakistan, Palestine, Säo Tomé \& Principe, Saudi Arabia, Seychelles, Spain, Somalia, South Africa, UA Emirates, Yemen, Zambia

Distribution in Egypt: Coastal Stripe, Lower Egypt

Type Locality: South Africa, Western Cap

Type specimens:

Holotype: Male, MNHN (Lost)

2. Condica conducta (Walker, 1857)

Caradrina conducta Walker, 1857

Perigea inexacta Walker, 1865

Condica decolorata (Guenée, 1862)

Perigea conducta (Walker, 1856)

Platysenta conducta (Walker, 1856)

Common name: Unknown

Wingspan: about $30 \mathrm{~mm}$

Host records: Senecio sp., Asteraceae

Geographical Records: Cape Verde, Comoros, Congo, Djibouti, Equatorial Guinea, Fiji, Hong Kong, India, Kenya, Madagascar, Malawi, Mauritania, Mauritius, Reunion, Seychelles, South Africa, Saudi Arabia, Yemen

Distribution in Egypt: Coastal Stripe

Type Locality: DRC (Democratic Republic of Congo) as Condica conducta

Type specimens:

Lecotype: Male (BMNH, London)

Paralectotype: 5 (Male \& Female) (BMNH, London)

3. Condica Palaestinensis (Staudinger, 1895)

Hadjina palaestinensis Staudinger, 1895

Common name: Unknown

Wingspan: 33 - $36 \mathrm{~mm}$

Host records: Grasses, Pluchea dioscorides

Geographical Records: Iran, Iraq, Israel, Pakistan, Palestine, Syria, Yemen

Distribution in Egypt: Sinai

Type Locality: Palestine/Israel, Jerusalem

Type specimens

Lecotype: female (ZMHB)

Prospalta coptica Wiltshire, 1948

4. Condica pauperata (Walker, 1858)

Hadena pauperata Walker, 1858

Common name: Unknown

Wingspan: 33 - $36 \mathrm{~mm}$

Host records: Asteraceae; (Grasses, Pluchea dioscorides; Bidens pilosa); Apiaceae 
(Caropsis sp.)

Geographical Records: Cape Verde, Comoros, DR Congo, Madagascar, Mauritius, Reunion, Saudi Arabia, Seychelles, Sierra Leone, South Africa, Säo Tomé \& Principe, Uganda, Yemen

Distribution in Egypt: Gebel Elba, Fayoum, Sinai, Western Desert

Type Locality: South Africa, KwaZulu-Natal, Port Natal [Durban], as Condica pauperata Egypt, Marsa Halaib, Gebel Elba, as Prospalta coptica

Type specimens

Holotype: Male (BMNH, London) as Condica pauperata

5. Condica viscosa (Freyer, 1831)

Hadjena viscosa Freyer, 1831

Hadjina indelicata Turati, 1934

Condica europaea Parenzan, 1980

Mythimna viscosa Freyer, 1831

Common name: Unknown

Wingspan: $25-29 \mathrm{~mm}$

Host records: Inula viscosa (Asteraceae)

Geographical Records: Albania, Algeria, Bulgaria, Crete, Croatia, Cyprus, Greece, Iran, Iraq, Israel, Italy, Jordan, Lebanon, Libya, Mauritania, Morocco, Oman, Portugal, Saudi Arabia, Sicily, Spain, Syria, Turkey, United Arab Emirates, Yemen

Distribution in Egypt: Sinai

Type Locality: Italy (Sicily)

\section{Subfamily Cuculliinae Herrich-Schäffer, [1850] (Plate II)}

Cuculliinae is a large and phylogenetically compact subfamily with more than 200 species worldwide (Speidel et al., 1996a). Most species are xerophilous, inhabiting steppes, eremic areas and/or xeromontane habitats (Ronkay \& Ronkay 1994, 1995). They are usually quite attracted to ultraviolet light, but often not before midnight.

Genus Cucullia Schrank, 1802 is comprised of some extremely similar species and so correct identification can sometimes be difficult. The Palearctic region contains more than 130 species (Ronkay \& Ronkay, 1994). Three different species-groups are clearly distinguished based on the selection of larval food-plants, on the female genitalia and on the appearance of the moths. These species-groups are either elevated to the status of separate genera (Ronkay \& Ronkay, 1994) or retained as valid subgenera (Fibiger \& Hacker, 2005). The larvae mainly feed on Asteraceae (Compositae) and Lamiaceae (Labiatae) (Ronkay \& Ronkay, 1994). 9 species belonging to 3 genera under one tribe were recorded in Egypt. Two types from Egypt, Cucullia hemidiaphana Graeser, 1892 type as (Cucullia efflatouni Wiltshire, 1948) and Cucullia macara Rebel, 1947.

Subfamily Cuculliinae Herrich-Schäffer, [1850]

Tribe Cucullini Ronkay \& Gyulai, 2011

1. Cucullia Schrank, 1802

1. Cucullia calendulae Treitschke, 1835

2. Cucullia chamomillae (Denis \& Schiffermüller, 1775)

3. Cucullia hemidiaphana Graeser, 1892

4. Cucullia macara Rebel, 1947

5. Cucullia melanoglossa (Berio, 1934)

6. Cucullia santolinae Rambur, 1834

7. Cucullia syrtana (Mabille, 1888)

2. Shargacucullia Ronkay \& Ronkay, 1992

8. Shargacucullia strigicosta (Boursin, 1940) 
9. Shargacucullia verbasci Linnaeus, 1758

\section{Metlaouia Dumont, 1929}

10. Metlaouia autumna (Chrétien, 1910)

Tribe: Cucullini

1. Cucullia Schrank, 1802

1. Cucullia calendulae Treitschke, 1835

Cucullia wredowi Costa, 1836 (Type; Italy)

Cucullia wredowi judaeorum Wiltshire, 1948

Cucullia dellabrunai Berio, 1980 (Holotype: Italy)

Cucullia leucanthemi Rambur, 1858 (Type: Spain)

Cucullia hermiguae Pinker \& Bacallado, 1979

Common name: Unknown

Wingspan: 40 - $46 \mathrm{~mm}$.

Host records: The larvae feed on Asteraceae, Chrysanthemum coronarium, (Glebionis

coronaria), Calendula, Ormenis, Achillea, Anthemis

Geographical Records: Afghanistan, Albania, Algeria, Croatia, Cyprus, England, France, Greece, Iran, Iraq, Israel, Italy, Jordan, Lebanon, Libya, Malta, Morocco, Palestine, Portugal, Saudi Arabia, Sicily, Spain, Syria, Tunisia, Turkey, Turkmenistan

Distribution in Egypt: Sinai

Type locality: Italy, Sicily

Type Specimens:

Lecotype: HNHM, Budapest

2. Cucullia chamomillae (Denis \& Schiffemüller, 1775)

Noctua chamomillae Denis \& Schiffermüller, 1775

Noctua fissina Haworth, 1809

Noctua chrysanthemi Hübner, [1822]

Cucullia residua Berio, 1992

Common name: Chamomile Shark, Chamomile monk

Wingspan: $43-50 \mathrm{~mm}$

Host records: Various Compositae, Chamaemelum, Achillea, Anthemis, Artemisia, Chrysanthemum, Matricaria

Geographical Records: Algeria, Austria, Belgium, Crete, Croatia, Czech Republic, Denmark, England, France, Germany, Greece, Hungary, Israel, Italy, Norway, Malta, Morocco, Palestine, Poland, Portugal, Romania, Sicily, Spain, Sweden, Switzerland, Syria, Tunisia, Turkey, Ukraine

Distribution in Egypt: Coast Stripe

Type Locality: Austria, Wien

Type specimen:

Type: Destroyed

3. Cucullia hemidiaphana Graeser, 1892

Cucullia efflatouni Wiltshire, 1948

Cucullia galleti Rungs, 1972

Common name: Unknown

Wingspan: $30-35 \mathrm{~mm}$

Host records: Artemisia sp.

Geographical Records: Afghanistan, Azerbaijan, Iran, Jordan, Kyrgyzstan, Morocco, Oman, Turkey, Turkmenistan, UA Emirates

Distribution in Egypt: Sinai

Type Locality: Egypt, S. Sinai, Wadi el Lega, Um Arab, Farsh el Arab as Cucullia efflatouni Kyrgyzstan, as Cucullia hemidiaphana 
Type specimen:

Type: Alfieri Coll. Cairo (Destroyed) as C. efflatouni

Paratype: Male (BMNH, London) C. efflatouni

Syntype: 2 Males and one male (ZMHB) as Cucullia hemidiaphana

4. Cucullia macara Rebel, 1947

Cucullia macara ssp. benderi Boursin, 1963

Cucullia leptographa Reisser, 1958

Common name: Unknown

Wingspan: about $32 \mathrm{~mm}$

Host records: L.T.

Geographical Records: Israel, Jordan, Palestine, Saudi Arabia

Distribution in Egypt: Gebel Elba, Sinai

Type Locality: Egypt, Gebel Elba, Wadi Cansisrob

Type specimens:

Syntype: Male \& Female (NHMW)

5. Cucullia melanoglossa (Berio, 1934)

Pseudocopicucullia melanoglossa Berio, 1934

Common name: Unknown

Wingspan:

Host records: L.T.

Geographical Records: Libya

Distribution in Egypt: Sheikh Fadl (Upper Egypt)

Type Locality:

Type specimens:

Syntype:

6. Cucullia santolinae Rambur, 1834

Cucullia amaenisima Oberthür, 1918

Common name: Holy Linae

Wingspan: $40-44 \mathrm{~mm}$

Host records: Artemisia, Santolina sp.

Geographical Records: Algeria, Corsica, France, Israel, Italy, Jordan, Lebanon, Morocco,

Palestine, Portugal, Sardinia, Sicily, Spain, Tunisia, Turkey

Distribution in Egypt: Gebel Elba

Type Locality: France, Corisca as Cucullia santolinae

Algeria, Biskra as Cucullia amaenisima

Type: BMNH, London as Cucullia amaenisima

7. Cucullia syrtana (Mabille, 1888)

Pseudocopicucullia syrtana subsp. iranica Brandt 1841

Common name: Unknown

Wingspan: $35-42 \mathrm{~mm}$

Host records: Asteraceae (Launaea sp.)

Geographical Records: Algeria, Cyprus, Greece, Iran, Iraq, Israel, Jordan, Kuwait, Malta, Mauritania, Libya, Morocco, Palestine, Saudi Arabia, Tunisia, UA Emirates

Distribution in Egypt: Lower Egypt, Costal Stripe, Eastern Desert, Sinai

Type Locality: Tunisia, Gebes

Type specimens:

Holotype: MNHN, Paris

2. Shargacucullia Ronkay \& Ronkay, 1992

8. Shargacucullia strigicosta (Boursin, 1940)

Cucullia strigicosta Boursin, 1940 
Common name: Unknown

Wingspan: $38-42 \mathrm{~mm}$.

Host records: Scrophularia sp, Artemisia monosperma, Retama raetam and Helianthemum

stipulatum

Geographical Records: Azerbaijan, Iran, Iraq, Israel, Jordan. Palestine, Turkey

Distribution in Egypt: Sinai

Type Locality: Iraq, (Near Khannikin, Kizil-Robat, Djebel Darawishka).

Type specimens:

Holotype: Male (LNK, Karlsruhe, Germany)

9. Shargacucullia verbasci Linnaeus, (1758)

Cucullia verbasci Linnaeus, 1758

Noctua verbasci Linnaeus, 1758

Cucullia oberthueri Rothschild, 1911

Common name: mullein moth

Wingspan: $45-55 \mathrm{~mm}$

Host records: Verbascum, Scrophularia, Celsia sp., Buddleia sp.; Artemisia monosperma

Geographical Records: Albania, Austria, Belgium, Bulgaria, Croatia, Cyprus, England, French, Germany, Hungary, Iran, Ireland, Israel, Italy, Jordan, Lebanon, Malta, Palestine, Portugal, Romania, Sicily, Spain, Switzerland, Syria, Turkmenistan, Ukraine

Distribution in Egypt: Lower Egypt

Type Locality: Europe

Type: LS, London

3. Metlaouia Dumont, 1929

10. Metlaouia autumna (Chrétien, 1910)

Metlaouia pberthuri Chrétien, 1909

Cucullia oberthuri Culot, 1909

Simyra oberthueri (Culot, 1910) (T.: Algerien, Aflou, BMNH, London)

Simyra fiorii (Turati, 1922) (Holot.: Libyen, Cirenaica, Tobruk)

Simyra chpakowskii (Chnéour, 1942 (Holot.: Tunesien, Kasserine)

Common name: Unknown

Wingspan: $35-40 \mathrm{~mm}$.

Host records: Scrophularia sp., Artemisia campestris

Geographical Records: Algeria, Iraq, Israel, Lebanon, Libya, Morocco, Palestine, Tunisia,

Turkey,

Distribution in Egypt: Sinai

Type Locality: Tunisia (Gafsa)

Type: MNHN, Paris

\section{Subfamily Eriopinae Herrich-Schäffer, [1851] (Plate II)}

Subfamily Eriopinae was previously either placed in Amphipyrinae, Hadeninae, or its own subfamily Eriopinae. Due to the complicated taxonomic histories of all the trifine groups of the Noctuidae sensu lato during the last decades, and the varying intensities of the evidence used for inferring the phylogenetic relationships, no consensus about the systematic position of the Eriopinae has ever been reached.

Callopistria, the type genus of the Eriopinae, was originally confined to its type species. It placed also in Acronictinae (as Acronictinae) and in Hadeninae as (Eriopus). Kitching (1984) reviewed the concepts of Noctuidae subfamilies and suggested placing Callopistria in the Amphipyrinae, which was considered by Kitching at least as paraphyletic. Poole (1995), suggested placing Callopistria and four other New World genera in the Eriopinae. Kitching \& Rawlins (1999) transfer Callopistria back into Hadeninae based on the 
superficial similarity in male genitalia and some plesiomorphic features of the pupa. Fibiger \& Lafontaine (2005) discussed the validity of Eriopinae and suggested adding a few more African genera; Fibiger \& Hacker (2007) further extended the taxonomic boundary of Eriopinae to accommodate the genus Prometopus Guenée.

So far, 2 species have been recorded in Egypt belonging to one genera.

Type genus: Eriopus Treitschke, 1825 (= Callopistria Hübner, [1821] 1816)

Subfamily Eriopinae Herrich-Schäffer, [1851]

1. Callopistria Hubner, [1821]

1. Callopistria latreillei (Duponchel, 1827)

2. Callopistria yerburii (Butler, 1884)

1. Callopistria Hubner, [1821]

1. Callopistria latreillei (Duponchel, 1827)

Noctua latreillei, Duponchel, 1827

Methorasa latreillei (Duponchel, [1828])

Callopistria roseitelum (Walker, 1858)

Callopistria anthracita (Wanger, 1926)

Eriopus rechingeri (Koutsaftikis, 1973

Common name: Latreille's Latin

Wingspan: $25-33 \mathrm{~mm}$

Host records: Pteridaceae (Adiantum sp.), Aspleniaceae (Ceterach officinarum), Cochlearia sp. Geographical Records: Albania, Algeria, Angola, Austria, Bulgaria, Cape Verde, Crete, Croatia, Cyprus, DR Congo, Ethiopia, France, Greece, Hungary, India, Iran, Iraq, Israel, Italy, Kenya, Reunion, Lesotho, Madagascar, Malta, Morocco, Oman, Pakistan, Palestine, Portugal, Romania, Sardinia, Saudi Arabia, Somalia, South Africa, Spain, Switzerland, Syria, Tanzania, Turkey, UA Emirates, Yemen, Zimbabwe

Distribution in Egypt: Lower Egypt, Sinai

Type Locality: France, Provence Bretagne

Type Specimens:

Syntype: France

2. Callopistria yerburii (Butler, 1884)

Callopistria promiscua, Saalmüller, 1891

Common name: Yerbur's Tangle

Wingspan: about $27 \mathrm{~mm}$

Host records: Pteridaceae (Adiantum sp.), Aspleniaceae (Asplenium sp.)

Geographical Records: Botswana, DR Congo, Eritrea, Eswatini, Ethiopia, India, Reunion, Lesotho, Madagascar, Mozambique, Oman, Saudi Arabia, Seychelles, Somalia, South Africa, Sri Lanka, Yemen, Zambia, Zimbabwe

Distribution in Egypt: Lower Egypt, Sinai

Type Locality: Yemen, Aden

Type specimens:

Holotype: Male, (BMNH, London)

\section{Subfamily Eustrotiinae Grote, 1882 (Plate II)}

Eustrotiinae were often included in the Acontiinae, but interpreted by Fibiger \& Lafontaine (2005) as an independent subfamily. It includes about 200 species worldwide (Speidel et al., 1996a). The systematic scope is very uncertain. The genus-group of Eublemma is included in the database "Butterflies and Moths of the World - Generic Names and their Type-species" of the Natural History Museum London. This group is described by Kravchenko et al. (2007) considered as a separate subfamily Eublemminae. The Eustrotiinae are probably paraphyletic. 
Up to date, 11 species were recorded in Egypt under 3 genera. There are 5 types from Egypt two of them (Ozarba capreolana Rebel, 1947 \& Ozarba fuscescens Rebel, 1947) recorded only from Egypt.

Subfamily Eustrotiinae Grote, 1882

1. Eulocastra Butler, 1886

1. Eulocastra alfierii (Wiltshire, 1948)

2. Eulocastra diaphora (Staudinger, 1878)

3. Eulocastra platizona (Lederer, 1869)

4. Eulocastra rex (Wiltshire, 1948)

5. Eulocastra tamsina Brandt, 1947

2. Ozarba Walker, 1865

6. Ozarba capreolana Rebel, 1947

7. Ozarba fuscescens Rebel, 1947

8. Ozarba scotrana Berio, 1940

9. Ozarba variabilis Berio, 1940

3. Pseudozarba Warren, 1913

10. Pseudozarba bipartita (Herrich-Schäffer, 1850)

11. Pseudozarba menozona (Hampson, 1910)

1. Eulocastra Butler, 1886

1. Eulocastra alfierii (Wiltshire, 1948)

Eulocastra alfierii erythra Wiltshire, 1948, (Holot. Saudi Arabia, BMNH, London)

Thalerastria alfierii Wiltshire, 1948

Common name: Unknown

Wingspan: about $16 \mathrm{~mm}$.

Host records: L.T.

Geographical Records: Oman, Saudi Arabia, United Arab Emirates, Yemen

Distribution in Egypt: Sinai.

Type Locality: Egypt, S. Sinai, Bir Isla, Wadi Isla, Karm Alam as O. alfierii

Type specimens

Holotype: Male (USNM, Washington)

2. Eulocastra diaphora (Staudinger, 1878)

Erastria diaphora Staudinger, 1878)

Thalerastria laticincta (Staudinger, 1898); (Synt.: Turkey, Mardin, MNHU, Berlin)

Metachrostis atribasalis (Hampson, 1896); (T.: Yemen, Aden, BMNH, London)

Thalpochares triangularis Warren, 1888

Common name: Unkown

Wingspan: $15-17 \mathrm{~mm}$

Host records: Conyza albida

Geographical Records: Afghanistan, Algeria, Armenia, India, Iran, Iraq, Israel, Jordan, Lebanon, Morocco, Oman, Pakistan, Palestine, Russia, Saudi Arabia, Spain, Syria, Turkey, United Arab Emirates, Yemen

Distribution in Egypt: Lower \& Upper Egypt, Eastern Desert, Sinai, Gebel Elba

Type locality: Turkey, Kerasdere

Type Specimen:

Holotype: Female (MNHU, Berlin)

Thalerastria rex Wiltshire, 1948

3. Eulocastra rex (Wiltshire, 1948)

Common name: Unkown

Wingspan: about $16 \mathrm{~mm}$.

Host records: L.T. 
Geographical Records: Yemen, Saudi Arabia.

Distribution in Egypt: Red Sea, Eastern Desert

Type locality: Yemen, Asia Mountain, Khamis Mushait

Type specimen:

Holotype: Male, BMNH, London

4. Eulocastra platizona (Lederer, 1869)

Madopa platizona Lederer, 1869

Eulocastra schah Bytinski-Salz \& Brandt, 1937

Common name: Unkown

Wingspan: about $17 \mathrm{~mm}$

Host records: L.T.

Geographical Records: Iran, Transcaucasia (USSR).

Distribution in Egypt: Lower Egypt

Type locality: USSR, Transcaucasie

Type specimen:

Type: Transcaucasie, (MNHU, Berlin)

Holotype: Iran, Keredj, male (NR, Stockholm) as E. schah

5. Eulocastra tamsina Brandt, 1947

Eulocastra tamsi Brandt, 1941

Common name: Unkown

Wingspan: $14-17 \mathrm{~mm}$.

Host records: L.T.

Geographical Records: Gambia, Iran, Oman, United Arab Emirates, Yemen

Distribution in Egypt: Upper Egypt, Gebel Elba

Type locality: Iran, Kouh, aftan: Bender Tchahbhar

Type specimen:

Syntype: NHRM, Stockholm

2. Ozarba Walker, 1865

6. Ozarba capreolana Rebel, 1947

Common name: Unknown

Wingspan:

Host records: L.T.

Geographical Records: Not Recorded

Distribution in Egypt: Gebel Elba

Type Locality: Egypt, Gebel Elba, Mersa Halaib

Type specimens

7. Ozarba fuscescens Rebel, 1947

Common name: Unknown

Wingspan:

Host records: L.T.

Geographical Records: Not Recorded

Distribution in Egypt: Gebel Elba

Type Locality: Egypt, Gebel Elba, Wadi Eideb

Type specimens

8. Ozarba socotrana Hampson, 1910

Ozarba cervina Rebel, 1948

Ozarba timida Berio, 1940

Common name: Unknown

Wingspan:

Host records: L.T. 
Geographical Records: Eritrea, Madagascar, Oman, Saudi Arabia, Yemen

Distribution in Egypt: Gebel Elba

Type Locality: Yemen, Socotra, Habidu Plain as Ozarba socotrana

Egypt, Gebel Elba, Wadi Eideb as Ozarba cervina

Eritrea, Elaberet, as Ozarba timida

Type specimens:

Holotype: Male, (BMNH, London) as Ozarba socotrana

Male, (MSNM) as Ozarba timida

Paratype: Male and Female (BMNH, London) as Ozarba timida

9. Ozarba variabilis Berio, 1940

Ozarba elephina Rebel, 1948

Common name: Unknown

Wingspan:

Host records: L.T.

Geographical Records: Eritrea, Ethiopia, Mauritania, Sudan, Tanzania, Yemen

Distribution in Egypt: Gebel Elba

Type Locality: Eritrea, Elaberet, as Ozarba variabilis

Egypt, Gebel Elba, Wadi Eideb as Ozarba elephina

Type specimens

Holotype: male, MSNM as Ozarba variabilis

2. Pseudozarba Waren in Seitz, 1913

10. Pseudozarba bipartita (Herrich-Schäffer, 1850)

Apamea bipartita Herrich-Schäffer, 1850

Pseudozarba pseudomorosa Hacker, 2016

Common name: Unknown

Wingspan: $16-20 \mathrm{~mm}$

Host records: Poaceae (Setaria sp. and others)

Geographical Records: Algeria, Bulgaria, Burkina Faso, Cape Verde, Croatia, Ethiopia, France, Gambia, Greece, Iran, Israel, Italy, Jordan, Kenya, Mauritania, Namibia, Niger, Nigeria, Palestine, Sicily, Somalia, South Africa, Spain, Sudan, Tanzania, Tunisia, Turkey, Yemen, Zambia,

Distribution in Egypt: Gebel Elba

Type Locality: Italy, Sicily

Type specimens

11. Pseudozarba mesozona (Hampson, 1910)

Eulocastra mesozona (Hampson, 1910)

Thalerastria mesozona Hampson, 1910)

Pseudozarba mediana (Staudinger, 1898)

Common name: Unknown

Wingspan: $15-19 \mathrm{~mm}$

Host records: LT.

Geographical Records: Djibouti, Eritrea, Gambia, Israel, Iran, Jordan, Oman, Palestine, UA

Emirates, Yemen.

Distribution in Egypt: Upper Egypt, Gebel Elba, Sinai

Type locality: Yemen, Aden

Type specimens:

Holotype: Female (BMNH, London) 


\section{Plate $T$}

Subfamily: Bryophilinae Guenée, 1852

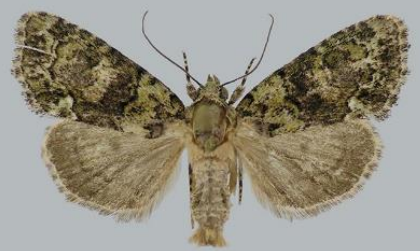

Cryphia algae (Fabricius, 1775

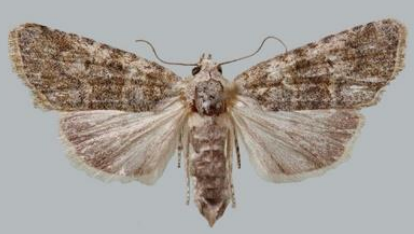

Cryphia moeonis (Lederer, 1865

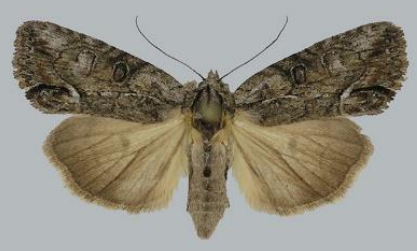

Cryphia raptricula (Denis \& Schiffermüller, 1775

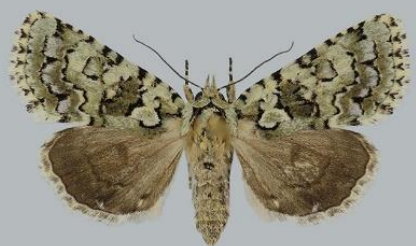

Cryphia amasina (Drandt, 193

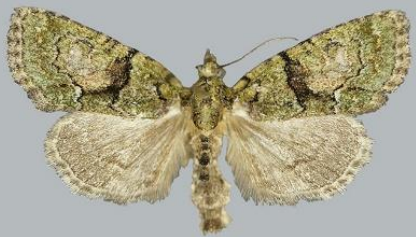

Cryphia pallida (Bethune-Baker, 1894

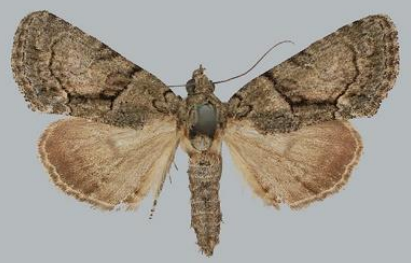

Cryphia receptricula (Hübner, 1803

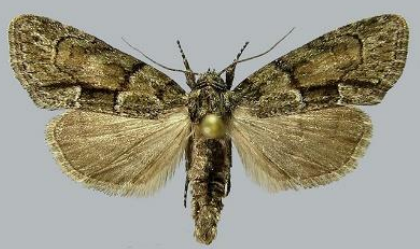

Cryphia fraudatricula (Hübner, 1803

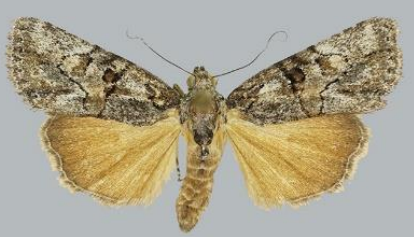

Cryphia petrea (Guenée, 1852

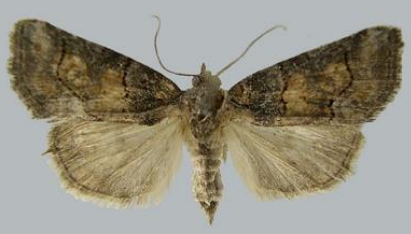

Cryphia vandalusiae (Duponchel, 1842

\section{Subfamily: Condicinae Poole, 1995}

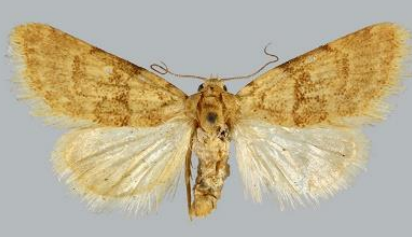

Acosmetia arida Joannis, 1909

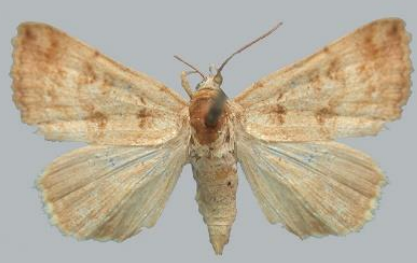

Condica Palaestinensis (Staudinger, 1895

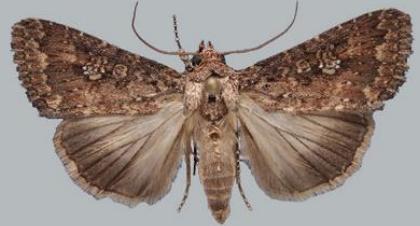

Condica capensis (Guenée, 1852

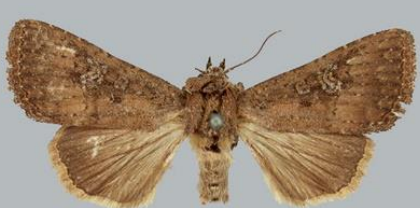

Condica pauperata (Walker, 1858

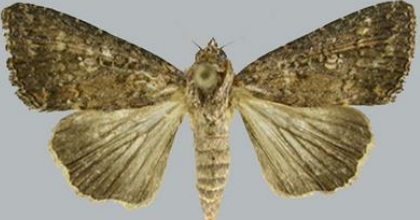

Condica conducta (Walker, 1857

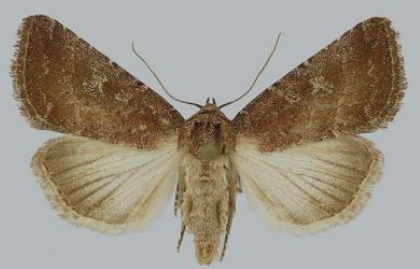

Condica viscosa (Freyer, 1831 


\section{Plate $T \mathcal{T}$}

Subfamily Cuculliinae Ferrich-Schäffer, 1845

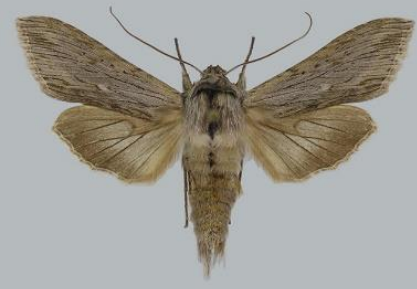

Cucullia calendula Treitschke, 1835

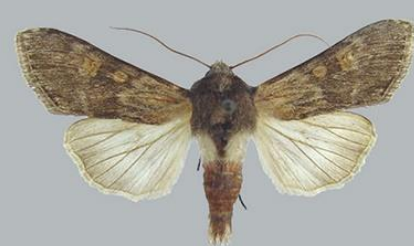

Cucullia macara Rebel, 1947

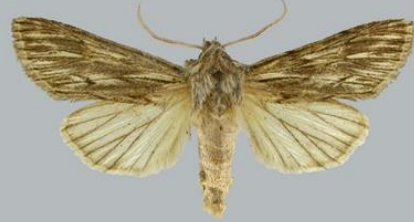

Metlaouia autumna (Chrétien, 1910

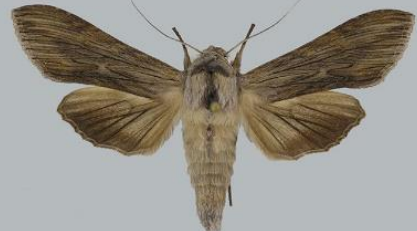

Cucullia chamomillae (Denis \& Schiffemüller, 1775

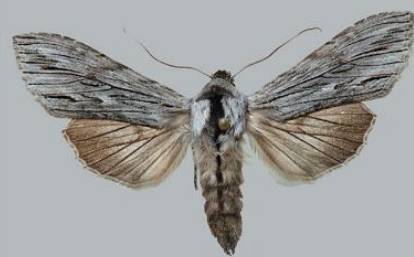

Cucullia santolinae Rambur, 1834

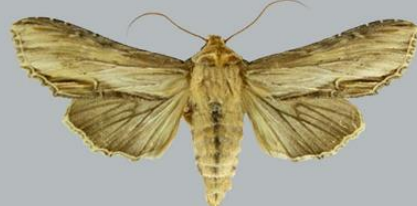

Shargacucullia strigicosta (Boursin, 1940

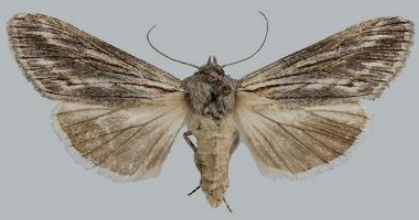

Cucullia hemidiaphana Graeser, 1892

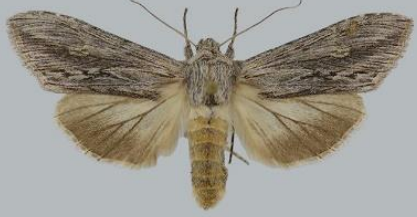

Cucullia syrtana (Mabille, 1888

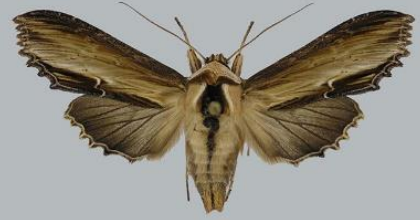

Shargacucullia verbasci Linnaeus, (1758

Subfamily Eriopinae Ferrich-Schäffer, [1851

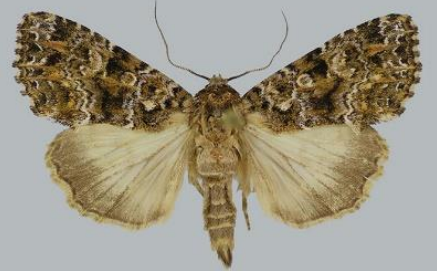

Callopistria latreillei (Duponchel, 1827

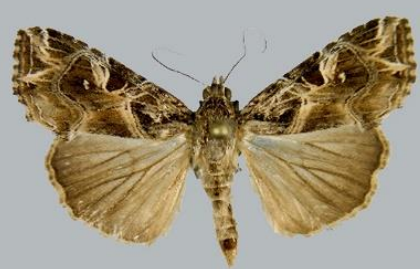

Callopistria yerburii (Butler, 188

Subfamily Eustrotiinae Grote, 1882

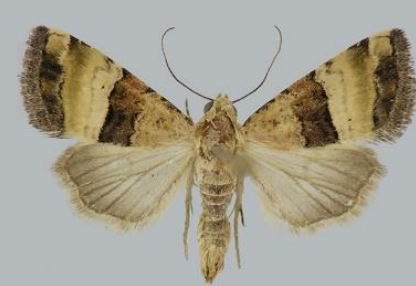

Pseudozabra bipartita (Herrich-Schäffer, 1850

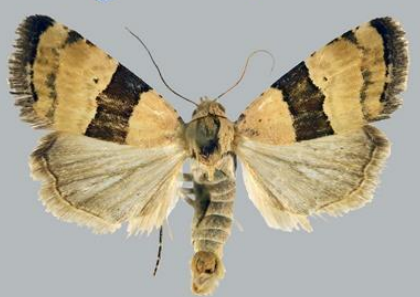

Pseudozabra mesozona (Hampson, 1910 


\section{REFERENCES}

Abdel Fattah M. Amer (2005): Ecological and Taxonomical studies of some Lepidoptera from Egypt with special References to family Noctuidae. Ph.D. Thesis, Department of Entomology, Faculty of Science, Cairo University.

Abdel Fattah M. Amer and Ashraf M. El-Torkey (2017): Revision of Higher Classification of Superfamily Noctuoidea (Lepidoptera) in Egypt, Egypt. Acad. J. Biolog. Sci., 10(1):55- 69

Abdel Fattah M. Amer, Rizk, M. A. \& Iskandar, A. K. (2006): Impact of Environment on the Diversity of Lepidopterous Insects in Three Egyptian Governorates. CATRINA (2009), 1(1): 81-88.

Adolf Andres (1910a): Observations sur quelques Lépidoptères nouveau ou peu connus d'Egypte. Bulletin de la Société Etomologique d'Egypt, pp. 21-23

Ahmet Omer Kocak and Muhabbet Kemal (2009): Diversity of the Lepidoptera in the World (DLW); Lepidoptera in Africa (LAF). Cesa Publications of African Lepidoptera nr. 25

Albert Legrain and Edward P. Wiltshire (1998): Provisional Checklist of the Macroheterocera [Lepidoptera] of the UAE TRIBULUS Vol. $8.2 \mathrm{pp}$. (5-8)

Bang-Haas A. (1912): Neue oder wenig bekannte palaearktische Makrolepidopteren V. Deutsche entomologische Zeitschrift, Iris 26(3):139-162, pl. 6.

Beccaloni G.W., Scoble M.J., Robinson G.S. \& Pitkin B. (2005): LepIndex: The Global Lepidoptera Names Index.

Bethune-Baker G. T. (1894): Notes on some Lepidoptera received from the neighbourhood of Alexandria. - Transactions of the entomological Society of London 1894: 33-51

Bisby FA, Roskov YR, Orrell TM, Nicolson D., Paglinawan LE, Bailly N., Kirk PM, Bourgoin T., Baillargeon G., Ouvrard D. (red.) (2011). "Species 2000 \& ITIS Catalog of Life: 2011 Annual Checklist." Species 2000: Reading, UK . Retrieved June 28, 2014.

Bugnion, C. (1837): Description de quatre nouvelles espèces de Lépidoptères de la Syrie et de l'Égypte. - Annales de la Société Entomologique de France 6: 439-444 + pl. 16th Paris (FG Levrault).

Draudt, M. (1910): Liste des Lépidoptères recueillis en Egypt par M.G. Ferrante. Bulletin de la Société Etomologique d'Egypt, pp. 96-97.

Fibiger M. and Hacker H. (2007): Noctuidae Europaeae. Vol. 9, Amphipyrinae, Condcinae, Eriopinae, Xyleninae (part). Entomological Press, Sorø, 410pp.

Fibiger, M, \& Lafontaine J.D. (2005): A review of the higher classification of the Noctuoidea (Lepidoptera) with special reference to the Holarctic fauna. Esperiana 11: 7-92.

Fryer J. C. F. (1912): The Lepidoptera of Seychelles and Aldabra, exclusive of the Orneodidae and Pterophoridae and of the Tortricina and Tineina. - Transactions of the Linnean Society of London (2)15(Zool.) (1):1-28; pl. 1.

Graeser, L. (1892): New Lepidoptera from Central Asia. - Berlin Entomological Journal 37 (3): 299-318.

Hacker H. H. (2001a): Fauna of the Nolidae and Noctuidae of the Levante with descriptions and taxonomic notes (Lepidoptera, Noctuoidea). Appendix: Revision genus Clytie Hübner, [1823]. - Esperiana 8:7-398, pls. 1-22.

Hacker H. H. (2016a): Systematic and illustrated catalogue of the Macroheterocera and Cossoidea Leach, [1815], Zygaenoidea Latreille, 1809, Thyridoidea Herrich-Schäffer, 1846 and Hyblaeoidea Hampson, 1903 of the Arabian Peninsula, with a survey of their 
distribution (Lepid.). - Esperiana 20(1):1-742; (2): 1-430.

Hacker, H. \& Fibiger, M. (2006). "Updated list of Micronoctuidae, Noctuidae (s.1.), and Hyblaeidae species of Yemen, collected during three expeditions in 1996, 1998 and 2000, with comments and descriptions of species." Esperiana Buchreihe zur Entomologie 12: 75-166.

Hacker H. H. \& Saldaitis A. (2010): Noctuidae of the Socotra Archipelago (Yemen) with notes on the fauna of the southern Arabian Peninsula (Lepidoptera, Noctuoidea). Esperiana Memoir 5:172-241.

Hacker H. H., Schreier H.-P. \& Aistleitner E. (2010): Noctuidae of Cape Verde Islands (Lepidoptera, Noctuidae). - Esperiana Memoir 5:7-96.

Hacker H.H.; Aulombard F.; Bischof A.; Bittermann J.; Fibiger M. \& Schreier H.P. (2001): Lepidoptera of Yemen Arab Republic, collected during three expeditions in 1996, 1998 and 2000. - Esperiana 8:597-632.

Hampson G. F. (1910e): Catalogue of the Lepidoptera Phalaenae in the collection of the British Museum (Nat. Hist.). X. Noctuidae. 10: i-xix, 1-829.

Hampson G. F. (1913d): Catalogue of the Lepidoptera Phalaenae in the collection of the British Museum (Nat. Hist.) XIII. Noctuidae.13:i-xiv, 1-609. Taylor \& Francis, London.

Heppner, J. B. (1991): Classification of Lepidoptera. Holarctic Lepid., 5(1) supplement 1: 1-148.

Innes Bey (1909): Résumé de travaux relatife en partie a la faune entomologique d'Egypte.

Bulletin de la Société Etomologique d'Egypt pp. 10-16

Joannis, P.J. (1910): Nouveau Lèpidoptères d'Egypte. Bulletin de la Société Etomologique d'Egypt, 1: 161-170.

Karrom, M. A. (1976): Revision of some Genera of the Family Noctuidae (Lepidoptera) inA.R. Egypt. PhD. Thesis, Dep. Ent., Fac. Sci., Ain Shams University.

Kitching, I.J. (1984): A historical review of the higher classification of the Noctuidae (Lepidoptera). Bulletin of the British Museum (Natural History), Entomology series 49(3): 153-234.

Kitching, I. J. and Rawlins, J. E. 1999 [1998]. The Noctuoidea. pp. 355-401. In KRISTENSEN, N. P. (Ed.). Lepidoptera: Moths and Butterflies. Volume 1: Evolution, systematics and biogeography. - Handbook of Zoology/Handbuch der Zoologie. 491 pp. Berlin/New York: Walter de Gruyter.

Kravchenko, V. D.; Orlova, O.; Fibiger, M.; Mooser, J.; Li, C. \& Müller, G. C. (2006): The Acronictinae, Bryophilinae, Hypenodinae and Hypeninae of Israel (Lepidoptera: Noctuidae) SHILAP Revta. lepid., 34 (135): 255-264.

Kravchenko V. D., Fibiger M., Hausmann A. \& Müller G. C. (2007b): The Lepidoptera of Israel. Volume 2. Noctuidae. 2:1-320, incl. pls. 1-38.

Lafontaine, J. D. \& Fibiger, M. (2006): Revised Higher Classification of the Noctuoidea (Lepidoptera). Bull. ent. Soc. Can., 138: 610-635.

Legrain A. \& Wiltshire E. P. (1998). Provisional checklist of the Macro-Heterocera (Lepidoptera) of the UAE. - Tribulus 8(2):5-8.

Lehmann L. (2010b): Records of interesting eremic Noctuidae species new to several southern Mediterranean countries (Lepidoptera, Noctuidae). - Esperiana Memoir 5:409-412, pl. 20.

Lehmann L. \& Saldaitis A. (2006): Beitrag zur Nachtfalter-Fauna des Sinai (Lepidoptera). Esperiana 12: 205-209, pls. 23, 24.

Mabille P., (1888): Description de quelques Lépidoptères nouveaux de Tunisie et d'Algérie (2ème partie). - Annales de la Société entomologique de France, sér. 6, 8: LI-LII. 
Ménétriès M. (1849): Catalogue des insectes recueillis par feu M. Lehmann avec les descriptions des nouvelles espèces (seconde et dernière partie). - Mémoires de l'Académie impériale des sciences de St.-Pétersbourg Série 6, vol. 8(2):218-328, pls. 3-6.

Oshaibah, A. A. (1981): Classification of some moths of family Noctuidae-Lepidoptera in Egypt. Ph.D. Thesis, Ent. Zool. Dept., Gac.Sci., Al-Azhar University.

Pekarsky, O. \& Ronkay, L. (2010): "Subfamily Bryophilinae Guenée. The Bryophila raptricula species-complex. in: Fibiger, M., Ronkay, L., Yela, J.L. \& Zilli, A. (2010): Rivulinae - Phytometrinae, and Micronoctuidae, including Supplement to Noctuidae Europaeae, vols 1-11. Noctuidae Europaeae, volume 12." - Entomological Press, Sor $\varnothing$, 504 pp. +13 colour plates.

Poole, R. W. (1989): Lepidopterorum Catalogus (N.S.), Fasc. 118, Noctuidae part 1, $2,3$. Leiden/ New York: E. J. Brill/ Flora \& Fauna Publications, xii + 1314pp.

Poole, R. W. (1995): Noctuioidea: Noctuidae (part), Cuculliinae, Stiriinae, Psaphidinae (Part). pp. 249. In: R. B. Dominick et al., eds. The Moths of America North of Mexico Fasc. 26. Washington, DC.: The Wedge Entomological Research Foundation.

Rebel H. (1909). In: Kneucker A., Zoologische Ergebnisse zweier in den Jahren 1902 und 1904 durch die Sinaihalbinsel unternommener botanischer Studienreise nebst zoologischen Beobachtungen aus Ägypten, Palästina und Syrien (Lepidoptera). Verhandlungen des naturwissenschftlichen Vereins Karlsruhe 21:129-137

Rebel H. (1948): New heteroceras from Egypt. - Journal of the Vienna Entomological Society 32 (1947) (5-7): 49-60

Ronkay, G. \& Ronkay, L. (1994): Noctuidae Europaeae, Cuculiinae I, Volume 6.

Ronkay, G. \& Ronkay, L. (1988): Taxonomic studies on the Palaearctic Cuculliae (Lepidoptera, Noctuidae), Part IV. Annales Histrico-Naturales Musei Nationalis Hungarici, 80: 91-103.

Ronkay, L., Ronkay, G. \& Behounek, G. (2008): Plusiinae 1. A Taxonomic Atlas of the Eurasian and North African Noctuoidea. Volume 1. - Heterocera Press, Budapest. 342 pp.

Ronkay, G. \& Ronkay, L. (2009): Cuculliinae 1. A Taxonomic Atlas of the Eurasian and North African Noctuoidea. Volume 2. - Heterocera Press, Budapest. 365 pp.

Treitschke, F. (1835): The Butterflies of Europe 10 (2): 1-340. Leipzig (Ernst Fleischer).

Seitz, A. (1938): The Macrolepidoptera of the World, Vol.3; The Palearctic Noctuid Moth

Seitz, A. [Ed.] (1931-1938): The Great Butterflies of the Earth. A systematic processing of the previously known large butterflies. Supplement to volume 3. The palaearctic owllike moths: I-VI, 1-332, pl. 1-26.

Speidel, W., Fänger, H. and Naumann, C. M. (1996a): The phylogeny of the Noctuidae (Lepidoptera). Systematic Entomology, 21: 219-251.

Storey, G., B.A., F.E.S. (1914): Notes on some Egyptian Lepidoptera. Bulletin de la Société Etomologique d'Egypt, pp. 73-75.

Storey, G., B.A., F.E.S. (1916): List of Egyptian insects in the Collection of the Ministry of Agriculture. Cairo, Government Press, 52pp.

Viette P. \& Fletcher D.S., (1968): The types of Lepidoptera Heterocera described by P. Mabille. - Bulletin of The British Museum (Natural History) Entomology, 21: 389-425.

Walker F. (1857a): List of the Specimens of Lepidopterous Insects in the Collection of the British Museum. Part X. Noctuidae. 10 (1856): i-iv, 253-491.

Walker F. (1870): A list of the Lepidoptera collected by J. K. Lord, Esq., in Egypt, along the African shore of the Red Sea, and in Arabia; with descriptions of the species new to science.- The Entomologist 5(80-81):123-134; (82): 151-155.

Wiltshire E. P. (1947b). Middle East Lepidoptera, VII: New species and forms from Egypt 
and Arabia. - Bulletin of the Society Fouad I Entomology 31(1):4-11.

Wiltshire E. P. (1948a): The Lepidoptera of the Kingdom of Egypt, part 1. - Bulletin of the Society Fouad I Entomology 32:203-294, pls. 1-8

Wiltshire E. P. (1977a). Middle East Lepidoptera XXXIV. More new species of Noctuidae (Lep.) from Africa and Arabia, with further records of Lepidoptera from the Sudan. Mitteilungen der Münchner Entomologischen Gesellschaft 66:127-140.

Wiltshire E. P. (1980a): Lepidoptera: Fam. Cossidae, Limacodidae, Sesiidae, Lasiocampidae, Sphingidae, Notodontidae, Geometridae, Lymantriidae, Nolidae, Arctiidae, Agaristidae, Noctuidae, Ctenuchidae. - In: Wittmer \& Büttiker (eds.), Fauna of Saudi Arabia 2. Fauna of Saudi Arabia 2:179-240.

Wiltshire E. P. (1988): Fam. Metarbelidae, Geometridae, Arctiidae, Agaristidae, Noctuidae (part 6). - In Büttiker, W. \& Krupp, F. (eds.) Fauna of Saudi Arabia. - Fauna of Saudi Arabia 9: 68-82.

Zerny H. (1915): Zwei neue paläarktische Noctuiden. - Verhandlungen der zoologischbotanischen Gesellschaft Wien 65:222-225.

* www. Bestimmungshilfe für die in Europa nachgewiesenen Schmetterlingsarten

* www.funet.fi/pub/sci/bio/life/.../lepidoptera/.../noctuidae/.../calopha...

* www.noctuidae.de (Noctuidae, Nolidae und Pantheidae von Bernd Schacht) 


\section{ANNEXE 1 \\ Recent situation of Noctuidae in Egypt}

After the revision of all specimens, collections and all references we found:

* Pseudohadena jordana (Staudinger, 1900) transfer from subfamily Hadeninae to subfamily Xyleninae

* Aegoceropsis brevivitta (Hampson, 1901) added to subfamily Agaristinae

* Prospalta coptica Wiltshire, 1948 is a synonomy to Condica pauperata (Walker, 1858)

(Condicinae)

* Both Cucullia syrtana (Mabille, 1888) and Cucullia verbasci Linnaeus, 1758 transfer to genus Shargacucullia (Cuculliinae)

* Anarta sodae (Boisduval, 1829) added to subfamily Hadeninae

* Aegle vespertalis Hübner, [1812] is a synonomy to Aegle semicana (Esper, 1798)

(Metoponiinae)

* Anarta sodae (Boisduval, 1829) added to subfamily Hadeninae

* Bryomima sinaica Wiltshire, 1948 is a synonomy to Calophasia siniaca (Wiltshire, 1948)

(Oncocnemidinae)

* Calophasia melanglossa Berio, 1934 (Oncocnemidinae) not recorded in Egypt (get out from former list).

* Spodoptera exempta (Walker, 1857) (Xyleninae) 1934 not recorded in Egypt (get out from former list).

* Caradrina flavirena Güenee, 1852 (Xyleninae) 1934 not recorded in Egypt (get out from former list).

* Caradrina apicimaculata Rebel, 1947 is a synonomy to Caradrina oberthuri (Rothschild, 1913) (Xyleninae)

* Luperina diversa (Staudinger, 1892) added to subfamily Xyleninae

* Conistra staudingeri Graslin, 1863 added to subfamily Xyleninae

* Pseudamathes volloni Lucas, 1907 added to subfamily Xyleninae

* Aporophyla australis (Boisduval, 1829) is a synonomy to Aporophyla nigra (Haworth, 1809) (Xyleninae)

* Ctenoplusia albostriata (Bremer \& Grey, 1853) added to subfamily Plusiinae (from Egyptian Nuba (Von F. Kasy, 1963)

* Ctenoplusia limbirena (Guenée, 1852) added to subfamily Plusiinae (from Egyptian Nuba (Von F. Kasy, 1963)

* Genus Eulocastra Butler, 1886 transfer from Acontiinae to Eustrodiinae

* There are 50 species as types (Types, Holotypes, Paratypes, ... etc)

* Family Noctuidae contain 16 subfamilies, 82 genera and 203 species as follows:

Summary of family Noctuidae in Egypt (Arranged according to the number of species)

\begin{tabular}{|l|l|c|c|}
\hline No. & \multicolumn{1}{|c|}{ Subfamily } & No. of Genera & No. of Species \\
\hline 1 & Xyleninae & 21 & 44 \\
\hline 2 & Noctuinae & 7 & 33 \\
\hline 3 & Hadeninae & 12 & 30 \\
\hline 4 & Acontiinae & 5 & 15 \\
\hline 5 & Oncocnemidinae & 8 & 15 \\
\hline 6 & Plusiinae & 8 & 12 \\
\hline 7 & Eustrotiinae & 3 & 11 \\
\hline 8 & Bryophilinae & 1 & 11 \\
\hline 9 & Cucullinae & 3 & 10 \\
\hline 10 & Condicinae & 2 & 6 \\
\hline 11 & Heliothinae & 4 & 5 \\
\hline 12 & Agaristinae & 2 & 3 \\
\hline 13 & Amphipyrinae & 2 & 3 \\
\hline 14 & Acronictinae & 1 & 2 \\
\hline 15 & Eriopinae & 1 & 2 \\
\hline 16 & Metoponiinae & 1 & 1 \\
\hline
\end{tabular}




\section{ANNEXE II List of Noctuidae type species in Egypt \\ Subfamily Bryophilinae Guenée, 1852 \\ 1. Cryphia minima Waltshire, 1949 from Wadi Hoff (Cairo, Lower Egypt) \\ 2. Cryphia pallida (Bethune-Baker, 1894) from Alexandria (Coastal Strip); (Type: BMNH) \\ 3. Cryphia vandalusiae (Duponchel, 1842) from Alexandria as Miana trilinea Bethune- Baker, 1894; (Type) \\ Subfamily Condicinae Poole, 1995 \\ 4. Acosmetia arida Joannis, 1909 from Mokattam, Cairo (Lower Egypt); (Type: MNHN) \\ 5. Condica pauperata (Walker, 1858) from Marsa Halaib, (Gebel Elba) as Prospalta coptica Wiltshire, 1948}

Subfamily Cuculliinae Herrich-Schäffer, [1850]

6. Cucullia hemidiaphana Graeser, 1892 from Wadi el Lega, Um Arab, Farsh el Arab (S. Sinai) as Cucullia efflatouni Wiltshire, 1948; (Type: Alfieri Collection, Destroyed; Paratype: BMNH)

7. Cucullia macara Rebel, 1947 from Wadi Cansisrob (Gebel Elba); (Syntype: NHMW) Subfamily Eustrotiinae Grote, 1882

8. Eulocastra alfierii (Wiltshire, 1948) from Bir Isla, Wadi Isla, Karm Alam (S. Sinai); (Holotype: USNM)

9. Ozarba capreolana Rebel, 1947 from Mersa Halaib (Gebel Elba)

10. Ozarba fuscescens Rebel, 1947 from Wadi Eideb (Gebel Elba)

11. Ozarba socotrana Hampson, 1910 from Wadi Eideb (Gebel Elba) as Ozarba cervina Rebel, 1948

12. Ozarba variabilis Berio, 1940 from Wadi Eideb (Gebel Elba) as Ozarba elephina Rebel, 1948

\section{Subfamily Acontiinae}

12. Acontia asbenensis (Rothschild, 1921) from Bir Tarfa, (S. Sinai) as Trache hemipentha Wiltshire, 1947; (Holotype: male, USNM, Washington; Paratype: male, BMNH, Lond.)

13. Acontia biskrensis Oberthur, 1887 from Gebel Elba as Tarache grisescens Rebel, 1947; (Syntype: Allfieri Coll., not found)

14. Acontia crassivalva (Wiltshire, 1947) from Sinai (Holotype: male, Alfieri collection (not found); USNM, Washington; genitalia in BMNH, London)

15. Acontia dichroa (Hampson, 1914) from Wadi Aideb (Gebel Elba) as Tarache seminigra Rebel, 1947, (Type)

16. Armada nilotica Bang-Hass, 1912, from Cairo (Lower Egypt); (Holotype: 2 males, MNHU, Berlin)

17. Epharmottomena eremophila (Rebrl, 1895); from Sinai as M. plumbizonata (Type: BMNH, London); from Cairo (Lower Egypt) as L. plumbizonata (Holotype: BMNH, London)

18. Pyraloides spodia Rebel, 1947; from Wadi Aideb (Gebel Elba); (Holotype: Alf. Coll.; male (not found))

\section{Subfamily Hadeninae}

19. Cardepia sociabilis (Graslin, 1850) from Alexandria (Coastal Stripe) as Mamestra afra Bethune-Baker, 1894; (Types: BMNH, London).

20. Hadula deserticola (Hampson, 1905) from Suez (Eastern Desert) as Aglossestra deserticola Hampson, 1905 (Holotype: BMNH, London)

21. Hadena syriaca (Osthelder, 1933) from Sinai as Anepia imitaria ssp. petroffi Wiltshire, 1948, (Holotype: BMNH, London) 
22. Thargelia gigantea Rebel, 1909, from W. Werdan, Ajun Mus Oasis, W, Werdan (Sinai) (Syntypes: NM, Vienna)

23. Mamestra immiscens Walker, 1870; from Sinai, (Type: Mount Sinai)

24. Mamestra mixtura Walker, 1870; from Sinai, (Type: Mount Sinai)

25. Mythimna languida (Walker, 1858) from Alexandria (Coastal Strip) as Mamestra abbas Bethune-Baker, 1894 and as as Aletia abbas Bethune-Baker, 1894 (Holotype: BMNH, London).

\section{Subfamily: Xyleninae}

26. Caradrina alfierii (Boursin, 1937) from Gebel Katherin (Sinai); (Holotype: female, coll. Alfieri; Paratype: Coll. Alfierii and BMNH, London).

27. Caradrina katherina Wiltshire, 1947 from Gebel Katherin (Sinai); (Holotype: Male, Coll. Alfieri; Allotype, female, Coll. Alfieri. Paratype: Coll. Alfieri, \& BMNH, London).

28. Scythocentropus inquinata (Mabille, 1888); from Cairo (Lower Egypt) as Centropodia ferrantei (Draudt, 1911).

29. Luperina grzimeki Hacker, 1989 from l-Agami (Costal Stripe); (Holotype: Male in Coll. Zoologische Staassammlung München)

30. Sidemia beduina Wiltshire, 1948 from AlArish (S. Sinai). (Holotype: USNM, Washington \& BMNH)

31. Sesamia cretica Lederer, 1857 from Al Arish (N. Sinai); (Type: MNHU, Berlin)

32. Sesamia wiltshirei Rungs, 1963; from Ismailia (Eastern Desert); (Holotype: MNHN, Paris).

33. Mniotype compitalis (Draudt, 1909) from Alexandria (Costal Strep); (Syntype: 2 males, destroyed).

34. Polymixis aurora (Turati, 1924) from Cairo (Lower Egypt) as Eumichtis aurora aegyptiaca Wiltshire, 1947; (Type: Coll. Alfierii, not found)

35. Xylena infusa Walker, 1870 from Gebel Elba; Type

36. Xylena lefebvrei Bugnion, 1837; Gebel Elba; Type

\section{Subfamily: Metoponiinae Herrich-Schäffer, 1851}

37. Aegle semicana (Esper, 1798) from Sinai as Aegle vespertalis form petroffi Andres \& Seitz, 1925; (Type: Coll. Alfierii, not found)

Subfamily Noctuinae Latreille, 1809

38. Agrotis alexandriensis Bethune-Baker, from Alexandria (Coastal Strip); (Syntypes: BMNH, London)

39. Agrotis inobtrusa Walker, 1870 from St. Kathrin, Tur Sinai, Mount Sinai (S. Sinai); (Holotype: Male, BMNH, London, probably lost).

40. Agrotis marginata Walker, 1870; from Mount Sinai, Wadi Genneh, (S. Sinai); (Holotype, male, coll. J. K. Lord, destroyed)

41. Agrotis pictifascia (Hampson, 1896) from Gebel Elba as Agrotis (Euxoa) pictifascia subsp. elabaensis Rebel, 1948; (Holotype: 3 females, NHMW, Vienna)

42. Agrotis pierreti (Bugnion, 1837) from Alexandria (Costal Stripe) (Syntypes: male \& female BMNH, London).

43. Agrotis trux (Hübner, [1824]) from Alexandria (Costal Stripe) as Agrotis trux nili Bethune-Baker, 1894; and from Mariout as Agrotis adolfi Corti \& Draudt, 1933; (Type: as A. nili, BMNH, London; Syntype, as A. adolfi 1 Male and 2 Female)

44. Euxoa wagneri Corti, 1926, from Dekela (Coastal Stripe) as Agrotis wagneri Corti, 1926; (Holotype: NM, Basel)

45. Feltia mollis (Walker, [1857]), from Mount Sinai (Sinai); (Holotype: male, coll. J. K. Lord destroyed) 
Subfamily Oncocnemidinae Forbes \& Franclemont, 1954

46. Calophasia sinaica Wiltshire, 1948; from Gebel Katherine, W. El Rabaa (S. Sinai); (Holotype: USNM, Washington)

47. Cerapoda aegyptiaca Joannis, 1910; from Helwan, Cairo (Lower Egypt); (Type: MNHN, Paris)

48. Metopoceras eutychina Rebel, 1948; from St. Katherine (S. Sinai); (Holotype: NHMW, Vienna)

49. Metopoceras kneuckeri (Rebel, 1903); from W. Esch-Schech and Serbal Mountain (Sinai); (Holotype: LNK, Karlsruhe)

50. Teinoptera culminifera Calberla, 1891; from Al Arish (N. Sinai); (Syntype: ZMHB, Hamburg) 


\section{ANNEXE III}

Recent List of Noctuidae Latreille, 1809 in Egypt

\section{Subfamily Acontiinae Guenée, 1841}

Tribe Acontiini Guenée, 1841

1. Acontia Ochsenheimer, 1816

1. Acontia asbenensis (Rothschild, 1921

2. Acontia biskrensis Oberthür, 1887

3. Acontia carnescens (Hampson, 1910)

4. Acontia crassivalva (Wiltshire, 1947)

5. Acontia dichroa (Hampson, 1914)

6. Acontia hortensis Swinhoe, 1884

7. Acontia karachiensis Swinhoe, 1889

8. Acontia lucida (Hufnagel, 1766)

9. Acontia opalinoides (Guenée, 1852)

Tribe Armadini Wiltshire, 1961

2. Armada Staudinger, 1884

10. Armada dentata (Staudinger, 1884)

11. Armada nilotica Bang-Haas, 1912

12. Armada panaceorum (Ménétriés, 1849)

3. Epharmottomena John, 1909

13. Epharmottomena eremophila (Rebel, 1895)

4. Iranada Wiltshire, 1977

14. Iranada turcorum (Zerny, 1915)

5. Pyraloides Rebel, 1947

15. Pyraloides spodia Rebel, 1947

2. Subfamily Acronictinae Heinemann, 1859

6. Simyra Ochsenheimer, 1816

16. Simyra albovenosa (Goeze, 1781)

17. Simyra dentinosa Freyer, 1839

3. Subfamily Agaristinae Herrich-Schäffer, [1858]

7. Aegoceropsis Karsch, 1895

17. Aegoceropsis rectilinea Boisduval, 1836

18. Aegoceropsis brevivitta (Hampson, 1901)

8. Heraclia Hübner, 1820

19. Heraclia geryon (Fabricius, 1781)

4. Subfamily Amphipyrinae Guenée, 1837

Tribe: Amphipyrini Guenée, 1837

9. Amphipyra Ochsenheimer, 1816

20. Amphipyra tetra (Fabricius, 1787)

21. Amphipyra tragopoginis (Clerck, 1759)

10. Matopo Distant, 1898

22. Matopo selects (Walker, 1865)

5. Subfamily Bryophilinae Guenée, 1852

11. Cryphia Hübner, 1818

23. Cryphia algae (Fabricius, 1775)

24. Cryphia amasina (Drandt, 1931)

25. Cryphia fraudatricula (Hübner, 1803)

26. Cryphia maeonis (Lederer, 1865)

27. Cryphia minima Waltshire, 1949 
28. Cryphia pallida (Bethune-Baker, 1894)

29. Cryphia paulina (Staudinger, 1892)

30. Cryphia petrea (Guenée, 1852)

31. Cryphia raptricula (Denis \& Schiffermüller, 1775)

32. Cryphia receptricula (Hübner, 1803)

33. Cryphia vandalusiae (Duponchel, 1842)

6. Subfamily Condicinae Poole, 1995

Tribe: Condicini Poole, 1995

12. Acosmetia Stephens, 1829

34. Acosmetia arida Joannis, 1909

13. Condica Walker, 1856

35. Condica capensis (Guenée, 1852)

36. Condica conducta (Walker, 1857)

38. Condica palaestinensis (Staudinger, 1895)

38. Condica pauperata (Walker, 1858)

39. Condica viscosa (Freyer, 1831)

7. Subfamily Cuculliinae Herrich-Schäffer, [1850]

Tribe Cucullini Ronkay \& Gyulai, 2011

14. Cucullia Schrank, 1802

40. Cucullia calendula Treitschke, 1835

41. Cucullia chamomillae (Denis \& Schiffermüller, 1775)

42. Cucullia hemidiaphana Graeser, 1892

43. Cucullia macara Rebel, 1947

44. Cucullia melanoglossa (Berio, 1934)

45. Cucullia santolinae Rambur, 1834

46. Cucullia syrtana (Mabille, 1888)

15. Shargacucullia Ronkay \& Ronkay, 1992

47. Shargacucullia strigicosta (Boursin, 1940)

48. Shargacucullia verbasci Linnaeus, 1758

16. Metlaouia Dumont, 1929

49. Metlaouia autumna (Chrétien, 1910)

8. Subfamily Eriopinae Herrich-Schäffer, [1851]

17. Callopistria Hubner, [1821]

50. Callopistria latreillei (Duponchel, 1827)

51. Callopistria yerburii (Butler, 1884)

\section{Subfamily Eustrotiinae Grote, 1882}

18. Eulocastra Butler, 1886

52. Eulocastra alfierii (Wiltshire, 1948)

53. Eulocastra diaphora (Staudinger, 1878)

54. Eulocastra platizona (Lederer, 1869)

55. Eulocastra rex (Wiltshire, 1948)

56. Eulocastra tamsina Brandt, 1947

19. Ozarba Walker, 1865

57. Ozarba capreolana Rebel, 1947

58. Ozarba fuscescens Rebel, 1947

59. Ozarba scotrana Berio, 1940

60. Ozarba variabilis Berio, 1940

20. Pseudozarba Warren, 1913

61. Pseudozarba bipartita (Herrich-Schäffer, 1850)

62. Pseudozarba menozona (Hampson, 1910) 
10. Subfamily Hadeninae Guenée, 1837

Tribe Hadenini Guenée, 1837

Subtribe: Discestrina Beck, 1996

21. Anarta Ochsenheimer, 1816

63. Anarta trifolii (Hufnagel, 1766)

64. Anarta sodae (Boisduval, 1829)

22. Cardepia Hampson, 1905

65. Cardepia affinis Rothschild, 1913

66. Cardepia irrisoria (Erschoff, 1998)

67. Cardepia sociabilis (Graslin, 1850)

23. Hadula Staudinger, 1889

68. Hadula deserticola (Hampson, 1905)

69. Hadula sabulorum (Alphéraky, 1882)

70. Hadula stoliczkana Moore, 1878

Subtribe: Hadenina Guenée, 1852

24. Enterpia Guenée, 1850

71. Enterpia laudeti (Boisduval, 1840)

25. Hadena Schrank, 1802

72. Hadena sancta Staudinger, 1840

73. Hadena silenes (Hübner, 1822)

74. Hadena silenides (Staudinger, 1895)

75. Hadena syriaca (Osthelder, 1933)

26. Odontelia Hampson, 19057

76. Odontelia daphnadeparisae Kravchenko et. al., 2007

27. Thargelia Pungeler, 1900

77. Thargelia gigantea Rebel, 1909

Subtribe: Mamestrina Beck, 1996

28. Haplocestra Aurivillius, 1910

78. Haplocestra similis Aurivillius, 1910

29. Hecatera Guenée, 1852

79. Hecatera dysodea ([Denis \& Schiffermüller], 1775)

80. Hecatera fixseni (Christoph, 1883)

81. Hecatera weissi (Draudt, 1934)

30. Mamestra Ochsenheimer, 1816

82. Mamestra immiscens Walker, 1870

83. Mamestra mixtura Walker, 1870

Tribe: Leucaniini Guenée, 1837

31. Leucania Ochsenheimer, 1816

84. Leucania herrichii Herrich-Schäffer, 1849

85. Leucania loreyi (Duponchel, 1827)

86. Leucania palaestinae Staudinger, 1897

87. Leucania punctosa (Treitschke, 1825)

88. Leucania putrescens (Hübner, 1824)

89. Leucania zeae Duponchel, 1827

32. Mythimna Ochsenheimer, 1816

90. Mythimna languida (Walker, 1858)

91. Mythimna sicula Treitschke, 1835

92. Mythimna unipuncta (Haworth, 1809)

11. Subfamily Heliothinae Biosduval, 1828

33. Helicoverpa Hardwick, 1965 
93. Helicoverpa armigera (Hübner, [1805])

34. Heliothis Ochsenheimer, 1816

94. Heliothis nubigera (Herrich-Schäffer, 1851)

95. Heliothis peltigera (Denis \& Schiffermüller, 1775)

35. Masalia Moore, 1881

96. Masalia albida (Hampson, 1905)

36. Schinia Hübner, 1818

97. Schinia scutosa (Denis \& Schiffermüller, 1715)

12. Subfamily3: Metoponiinae Herrich-Schäffer, 1851

37. Aegle Hübner, 1823

98. Aegle semicana (Esper, 1798)

13. Subfamily Noctuinae Latreille, 1809

Tribe Noctuini Latreille, 1809

Subtribe Agrotina Rambur, 1848

38. Agrotis Ochsenheimer, 1816

99. Agrotis alexandriensis Bethune-Baker, 1894

100. Agrotis bigramma (Hübner, 1803)

101. Agrotis catalaunensis (Millière, 1873)

102. Agrotis haifae Staudinger, 1897

103. Agrotis herzogi (Rebel, 1911)

104. Agrotis inobtrusa Walker, 1870

105. Agrotis ipsilon (Hufnagel, 1766)

106. Agrotis lasserrei (Oberthür, 1881)

107. Agrotis lata Treitschke, 1835

108. Agrotis marginata Walker, 1870

108. Agrotis pictifascia (Hampson, 1896)

110. Agrotis pierrettii (Bugnion, 1837)

111. Agrotis puta (Hübner, [1803])

112. Agrotis ripae (Hübner, [1823])

113. Agrotis sardzeana Brandt, 1941

114. Agrotis segetum (Denis \& Schiffermüller, 1775)

115. Agrotis spinifera (Hubner, 1808)

116. Agrotis trux (Hübner, [1824])

39. Dichagyris Lederer, 1857

117. Dichagyris erubescens (Staudinger, 1892)

118. Dichagyris flammatra (Denis \& Schiffermüller, 1775)

119. Dichagyris forficula (Staudinger, 1877)

120. Dichagyris imperator (Bang-Hass, 1912)

121. Dichagyris leucomelas Brandt, 1941

122. Dichagyris melanuroides (Kozhantschikov, 1930)

123. Dichagyris renigera (Hübner, [1808])

40. Euxoa Hübner, 1821

124. Euxoa anarmodia (Staudinger, 1897)

125. Euxoa canariensis diamondi Boursin, 1940

126. Euxoa nigrofusca (Esper, 1788)

127. Euxoa wagneri Corti, 1926

41. Feltia Walker, 1856

128. Feltia mollis (Walker, [1857])

Subtribe Noctuina Latreille, 1809

42. Noctua Linnaeus, 1758 
129. Noctua pronuba (Linnaeus, 1758)

43. Rhyacia Hübner, 1821

130. Rhyacia arenacea (Hampson, 1907)

44. Xestia Hübner, 1818

131. Xestia c-nigrum (Linnaeus, 1758)

14. Subfamily Oncocnemidinae Forbes \& Franclemont, 1954

45. Brachygalea Hampson, 1906

132. Brachygalea albolineata (Blachier, 1905)

46. Calophasia Stephens, 1829

133. Calophasia angularis Chrétien, 1911

134. Calophasia siniaca (Wiltshire, 1948)

47. Cerapoda Smith, 1894

135. Cerapoda aegyptiaca Joannis, 1910

48. Cleonymia Berio, 1966

136. Cleonymia chabordis (Oberthür, 1876)

137. Cleonymia jubata Oberthür, 1890

138. Cleonymia warionis (Oberthür, 1876)

49. Lithophasia Staudinger, 1892

139. Lithophasia quadrivirgula (Mabille, 1888)

50. Metopoceras Guenée, 1850

140. Metopoceras eutychina Rebel, 1947

141. Metopoceras khalildja Oberthür, 1884

142. Metopoceras kneuckeri (Rebel, 1903)

143. Metopoceras omar Oberthür, 1887

144. Metopoceras solituda (Brandt, 1938)

51. Rhabinopteryx Christoph, 1889

145. Rhabinopteryx subtilis (Mabille, 1888)

52. Teinoptera Calberla, 1891

146. Teinoptera culminifera Calberla, 1891

15. Subfamily Plusiinae Boisduval, [1828]

Tribe Argyrogrammatini Eichlin \& Cunningham, 1978

53. Argyrogramma Hübner, [1821]

147. Argyrogramma signata (Fabricius, 1775)

54. Chrysodeixis Hübner, [1821]

148. Chrysodeixis chalcites (Esper, 1789)

149. Chrysodeixis eriosoma (Doubleday, 1843)

55. Thysanoplusia Ichinose, 1973

150. Thysanoplusia circumscripta (Freyer, 1831)

151. Thysanoplusia daubei (Boisduval, 1840)

152. Thysanoplusia orichalcea (Fabricius, 1775)

56. Trichoplusia McDunnough, 1944

153. Trichoplusia ni (Hübner, 1803)

Tribe Plusiini Boisduval, [1828]

Subtribe: Euchalciina Chou \& Lu, 1979

57. Euchalcia Hübner, [1821]

154. Euchalcia maria (Staudinger, 1892)

Subtribe: Plusiina Boisduval, [1828]

58. Autographa Hübner, [1821]

155. Autographa gamma (Linnaeus 1758)

59. Ctenoplusia Dufay, 1970 
156. Ctenoplusia albostriata (Bremer \& Grey, 1853)

157. Ctenoplusia limbirena (Guenée, 1852)

60. Syngrapha Hübner, [1821]

158. Syngrapha circumflexa (Linnaeus, 1767)

16. Subfamily Xyleninae Guenée, 1837

Tribe Prodeniini Forbes, 1954

61. Spodoptera Guenée, 1852

159. Spodoptera cilium (Guenée, 1852)

160. Spodoptera exigua (Hübner, 1808)

161. Spodoptera littoralis (Boisduval, 1833)

Tribe Caradrinini Boisduval, 1840

Subtribe Caradrinina Boisduval, 1840

62. Caradrina Ochsenheimer, 1816

162. Caradrina alfierii (Boursin, 1937)

163. Caradrina aspersa (Rambur, 1834)

164. Caradrina atriluna Guenée, 1852

165. Caradrina bodenheimeri Amsel, 1935

166. Caradrina clavipalpis (Scopoli, 1763)

167. Caradrina flava Oberthür, 1876

168. Caradrina ingrata (Staudinger, 1897)

169. Caradrina katherina Wiltshire, 1947

170. Caradrina melanurina (Staudinger, 1901)

171. Caradrina oberthuri (Rothschild, 1913)

172. Caradrina scotoptera (Püngeler, 1914)

173. Caradrina selini Boisduval, 1840

174. Caradrina soudanensis (Hampson, 1918)

63. Catamecia Staudinger, 1898

175. Catamecia minima (Swinhoe, 1889)

64. Hoplodrina Boursin, 1937

176. Hoplodrina ambigua (Denis \& Schiffermüller, 1775)

65. Pseudoligia Staudinger, 1901

177. Pseudoligia similiaria (Ménétriés, 1849)

66. Scythocentropus Speiser, 1902

178. Scythocentropus inquinata (Mabille, 1888)

Tribe Cosmiini Guenée, 1852

Subtribe Cosmiina Guenée, 1852

67. Atethmia Hübner, [1821]

179. Atethmia ambusta (Denis \& Schiffermüller, 1775)

180. Atethmia centrago (Haworth, 1809)

Tribe Phlogophorini Hampson, 1918

68. Euplexia Stephens, 1829

181. Euplexia lucipara (Linnaeus, 1758)

69. Phlogophora Treitschke, 1825

182. Phlogophora meticulosa (Linnaeus, 1758)

Tribe Apameini Guenée, 1841

Subtribe Apameina Boisduval, 1828

70. Luperina Boisduval, 1829

183. Luperina diversa (Staudinger, 1892)

184. Luperina grzimeki Hacker, 1989

71. Nonagria Ochsenheimer, 1816 
185. Nonagria typhae (Thunberg, 1784)

72. Sidemia Staudinger, 1892

186. Sidemia beduina Wiltshire, 1948

Subtribe Sesamiima Fibiger \& Goldstein, 2005

73. Sesamia Guenée, 1852

187. Sesamia cretica Lederer, 1859

188. Sesamia nonagrioides (Lefebuvre, 1827)

189. Sesamia wiltshirei Rungs, 1963

6. Tribe Xylenini Guenée, 1837

Subtribe Antitypina Forbes \& Frandemont, 1954

74. Aporophyla Guenée, 1841

190. Aporophyla nigra (Haworth, 1809)

75. Boursinia Brandt, 1938

191. Boursinia discordans (Boursin, 1940)

192. Boursinia lithoxylea (Bang-Haas, 1912)

76. Conistra Hübner, [1821]

193. Conistra staudingeri Graslin, 1863

77. Mniotype Franclemont, 1941

194. Mniotype compitalis (Draudt, 1909)

78. Polymixis Hübner, [1820]

195. Polymixis aurora (Turati, 1924)

196. Polymixis juditha (Staudinger, 1898)

197. Polymixis lea (Staudinger, 1897)

198. Polymixis variabile (Stertz, 1915)

79. Pseudamathes Hampson, 1910

199. Pseudamathes volloni Lucas, 1907

80. Pseudohadena Alphéraky, 1889

200. Pseudohadena jordana (Staudinger, 1900)

81. Xanthia Ochsenheimer, 1816

201. Xanthia ocellaris (Borkhausen, 1792)

Subtribe Xylenina Guenée, 1837

82. Xylena Ochsenheimer, 1816

202. Xylena infusa Walker, 1870

203. Xylena lefebvrei Bugnion, 1837 